\title{
Almost structure-preserving analysis for weakly linear damping nonlinear Schrödinger equation with periodic perturbation
}

\author{
Weipeng $\mathrm{Hu}{ }^{1,2,3, *}$, Zichen Deng ${ }^{1,2}$, Tingting Yin ${ }^{1}$ \\ 1 School of Mechanics, Civil Engineering and Architecture, Northwestern Polytechnical \\ University, Xi'an, Shaanxi, 710072, P. R. China \\ 2 State Key Laboratory of Structural Analysis of Industrial Equipment, Dalian University of \\ Technology, Dalian, Liaoning, 116023, P. R. China \\ 3 State Key Laboratory of Solidification Processing, Northwestern Polytechnical University, \\ Xi'an, Shaanxi, 710072, P. R. China

\begin{abstract}
Exploring the dynamic behaviors of the damping nonlinear Schrödinger equation (NLSE) with periodic perturbation is a challenge in the field of nonlinear science, because the numerical approaches available for damping-driven dynamic systems may exhibit the artificial dissipation in different degree. In this paper, based on the generalized multi-symplectic idea, the local energy/momentum loss expressions as well as the approximate symmetric form of the linearly damping NLSE with periodic perturbation are deduced firstly. And then, the local energy/momentum losses are separated from the simulation results of the NLSE with small linear damping rate less than the threshold to insure structure-preserving properties of the scheme. Finally, the breakup process of the multisoliton state is simulated and the bifurcation of the discrete eigenvalues of associated Zakharov-Shabat spectral problem is obtained to investigate the variation of the velocity as well as the amplitude of the solitons during the splitting process.
\end{abstract}

Keywords: damping nonlinear Schrödinger equation; generalized multi-symplectic; breakup of multisoliton state; structure-preserving; periodic perturbation

\section{Introduction}

To embody the volatility in the classical mechanics model for the quantum system, E.

* Corresponding author: Tel: +86-29-88460403, Fax:+86-29-88460403, Email: wphu@ @wpu.edu.cn (Weipeng Hu) Page 1 of 26 
Schrödinger proposed a partial differential equation (PDE) to describe the time evolution of the quantum states for the physical system in 1926 [1], which was named as Schrödinger equation and initiated the quantum mechanics. In the past several decades, there were a large number of contributions concentrated on the solutions and the dynamic behaviours of some kinds of the Schrödinger equation [2-16].

A. O. Caldeira and A. J. Leggett moved the conservative structure of microscopic quantum mechanics into the dissipative macroscopic domain in 1983 [17]. M. D. Kostin showed the existence of the term added into the Hamiltonian of the time-dependent Schrödinger equation to remove energy without changing the wave-function normalization [18]. Unfortunately, the simulation on the dissipative quantum mechanics is difficult, in that, the rigorous treatment of dissipative term in the quantum domain needs to consider a large number of degrees of freedom, each of which is governed by a dissipative PDE [19]. Thus, to avoid the onerous numerical computation, most of the current studies on the damping nonlinear Schrödinger equation (NLSE) focused on the qualitative properties by various analytical methods [20-25].

The representative jobs on the numerical analysis of damping NLSE include: L. S. Peranich revealed the reason for that the occurrence of the sawteeth oscillations superimposed on the smooth wave solutions of the numerical solutions presented by M. Delfour, M. Fortin and G. Payr [26] for the linear damping NLSE [27]; F. Y. Zhang and S. J. Lu analysed the long-time stability and the convergence of some finite difference schemes for the weakly damping Schrödinger equation, and proved the existence of global attractor [28, 29]; W. Z. Bao and D. Jaksch introduced an extension of the time-splitting sine-spectral method for solving damping NLSEs and proved that the numerical method proposed was explicit, unconditionally stable, and time transversal invariant [30]; M. Asadzadeh investigated a streamline diffusion-based discontinuous Galerkin approximation for the numerical solution of the coupled damping NLSEs and extended the resulting method to a multiscale variational scheme [31].

But, avoiding or reducing the artificial dissipation in the simulation even for low-dimensional dissipative PDEs is a challenge for numerical methods. In this field, the symplectic schemes for Birkhoffian systems [32] and the conformal symplectic integrators [33] may result in some numerical schemes for the damping NLSE with tiny artificial dissipation. Focusing on the local geometric properties of the system, an almost structure-preserving strategy 
with tiny artificial dissipation will be proposed to investigate the dynamic behaviours of the following perturbed damping NLSE in this work,

$$
i \partial_{t} u+\alpha \partial_{x x} u+\beta|u|^{2} u+i \gamma u=h(x, t)
$$

where, $u$ is a complex wave function, $i=\sqrt{-1}, \alpha, \beta, \gamma$ are real constants, $h(x, t)$ is the external small perturbation considered as a periodic function of time $t$ and coordinate $x$.

According to the physical situations where the NLSE applied, the damping rate $\gamma$ can be classified as two types [34]: One is the collisional damping that will be considered in this paper, where the damping is independent of the wave number and the damping rate $\gamma$ can be assumed as a small positive number; another is the Landau damping, on a Maxwellian electron distribution, of Langmuir waves, the specific form of which was presented in Ref. [35] and limitations of which were pointed out in Ref. [34].

In the case of interactions between protein molecules and a weak laser field, the small periodic perturbation is proportional to the first-order derivative of the wave function with respect to coordinate $x$, hence, it can be formulated as $h(x, t)=\eta \partial_{x} u e^{i\left(k_{0} x-\omega_{0} t\right)}$, where $\eta$ is a small real constant, $k_{0}, \omega_{0}$ are real constants. For the propagating soliton excited by a vertical oscillation, the small periodic perturbation is directly proportional to the wave function, therefore, it can be formulated as $h(x, t)=\eta u e^{i\left(k_{0} x-\omega_{0} t\right)}$ [21]. The dynamic behaviours considered in this work are associated with the propagation of the multisoliton. At the same time, the handling processes of these two cases in the numerical method employed in this paper are similar. Thus, we will just focus on the latter case in this paper.

For the conservative NLSE without external perturbation, there were various numerical methods developed in the last century. Among which, the multi-symplectic method proposed by T. Bridges in 1997 [36] is remarkable, because it has paid great attention to the local geometric properties as well as the high-accuracy of the numerical schemes for the conservative NLSE in the Hamiltonian framework: T. Bridges presented the multi-symplectic structure and several local conservation laws of the NLSE based on the variational principle, which initiated the numerical investigation on the local geometric properties of the completely integrable NLSE [36]; furthermore, A. L. Islas and C. M. Schober developed a multi-symplectic scheme for the 1D 
NLSE and investigated the conservative properties of which [37]; J. Q. Sun and M. Z. Qin derived a six-point scheme for the coupled 1D NLSE, and proved that it is equivalent to the multi-symplectic Preissmann integrator [38]; subsequently, A. Aydin and B. Karasozen generalized the results presented in Ref. [38] to the integration of coupled NLSEs with periodic plane wave solutions [39]; J. L. Hong and his collaborators applied the Runge-Kutta-Nyström (RKN) methods to NLSEs with variable coefficients and proved that the scheme constructed by RKN method is multi-symplectic [40]; Recently, S. H. Song et al proposed the semi-explicit multi-symplectic splitting method [41] and the multi-symplectic wavelet collocation method [42] for NLSE.

For the introduction of the damping term into the NLSE, the numerical method employed should without artificial dissipation or just with tiny artificial dissipation. In our precious jobs [43, 44], we have developed the generalized multi-symplectic method with tiny artificial dissipation to record the dissipative effect in each time step and investigate the local dynamic behaviours of the weak damping dynamic systems. In this paper, the generalized multi-symplectic method will be employed to study the local dynamic behaviours of the damping NLSE with small periodic perturbation, including the losses of energy/momentum and the breakup phenomena of the multisoliton state, which will broaden the applied range of the generalized multi-symplectic method to the damping complex PDE systems.

\section{Generalized multi-symplectic formulations of damping NLSE}

The multi-symplectic form of the conservative NLSE has been derived from the Noether symmetry theory in 1997 [36], which can be generalized to the damping NLSE (1) naturally. In this section, the generalized multi-symplectic conservation law and the modified local conservation laws of energy/momentum will be deduced in detail. In this way, the effects of the damping and the periodic perturbation on the approximate local conservation laws of the NLSE (1) can be formulated explicitly. With these approximate local conservation laws, the structure-preserving characteristics of the numerical scheme constructed in the next section can be verified in the numerical experiments.

To transform the damping NLSE (1) from complex PDE to real coupled PDEs, rewrite the

periodic perturbation term as $h(x, t)=\eta u e^{i\left(k_{0} x-\omega_{0} t\right)}=\eta u\left[\cos \left(k_{0} x-\omega_{0} t\right)+i \sin \left(k_{0} x-\omega_{0} t\right)\right]$ and let 
$u=p+i q$, then,

$$
\left\{\begin{array}{l}
-\partial_{t} q+\alpha \partial_{x x} p+\beta\left(p^{2}+q^{2}\right) p-\gamma q=\eta\left[p \cos \left(k_{0} x-\omega_{0} t\right)-q \sin \left(k_{0} x-\omega_{0} t\right)\right] \\
\partial_{t} p+\alpha \partial_{x x} q+\beta\left(p^{2}+q^{2}\right) q+\gamma p=\eta\left[p \sin \left(k_{0} x-\omega_{0} t\right)+q \cos \left(k_{0} x-\omega_{0} t\right)\right]
\end{array}\right.
$$

Reducing the above second-order PDEs (2) to the following first-order PDEs with intermediate variables $\partial_{x} p=v, \partial_{x} q=w$,

$$
\left\{\begin{array}{l}
-\partial_{t} q+\alpha \partial_{x} v+\beta\left(p^{2}+q^{2}\right) p-\gamma q=\eta\left[p \cos \left(k_{0} x-\omega_{0} t\right)-q \sin \left(k_{0} x-\omega_{0} t\right)\right] \\
\partial_{t} p+\alpha \partial_{x} w+\beta\left(p^{2}+q^{2}\right) q+\gamma p=\eta\left[p \sin \left(k_{0} x-\omega_{0} t\right)+q \cos \left(k_{0} x-\omega_{0} t\right)\right] \\
\partial_{x} p=v \\
\partial_{x} q=w
\end{array}\right.
$$

which can be rewritten as matrix form,

$$
\mathbf{M} \partial_{t} \mathbf{z}+\mathbf{K} \partial_{x} \mathbf{z}=\nabla_{\mathbf{z}} S(\mathbf{z})+\boldsymbol{\varepsilon}(p, q, x, t)
$$

where

$$
S(\mathbf{z})=\frac{1}{2}\left[\frac{\beta}{2}\left(p^{2}+q^{2}\right)^{2}+\alpha\left(v^{2}+w^{2}\right)\right] \quad ; \quad \mathbf{z}=[p, q, v, w]^{\mathrm{T}}
$$

$\boldsymbol{\varepsilon}(p, q, x, t)=\left[-\gamma q-\eta p \cos \left(k_{0} x-\omega_{0} t\right)+\eta q \sin \left(k_{0} x-\omega_{0} t\right), \gamma p-\eta p \sin \left(k_{0} x-\omega_{0} t\right)-\eta q \cos \left(k_{0} x-\omega_{0} t\right), 0,0\right]^{\mathrm{T}}$

is an addition item comparing with the standard multi-symplectic form of the conservative NLSE that contains the linear damping and the periodic perturbation; $\mathbf{M}, \mathbf{K}$ are skew-symplectic matrices.

$$
\mathbf{M}=\left[\begin{array}{cccc}
0 & 1 & 0 & 0 \\
-1 & 0 & 0 & 0 \\
0 & 0 & 0 & 0 \\
0 & 0 & 0 & 0
\end{array}\right], \mathbf{K}=\left[\begin{array}{cccc}
0 & 0 & -\alpha & 0 \\
0 & 0 & 0 & -\alpha \\
\alpha & 0 & 0 & 0 \\
0 & \alpha & 0 & 0
\end{array}\right]
$$

Different from the generalized multi-symplectic forms those we presented for the damping systems considered in Refs. [43-46], the linear damping rate isn't contained in the coefficient matrices of Eq. (4), but in the addition term $\boldsymbol{\varepsilon}(p, q, x, t)$.

Following the outline of the deducing process for the multi-symplectic conservation law [36], the generalized multi-symplectic conservation law for the matrix form (4) can be expressed as,

$$
\partial_{t}(\mathrm{~d} p \wedge \mathrm{d} q)+\alpha \partial_{x}(\mathrm{~d} p \wedge \mathrm{d} v+\mathrm{d} q \wedge \mathrm{d} w)=\mathrm{d} \mathbf{z} \wedge \mathrm{d} \boldsymbol{\varepsilon}(p, q, x, t)
$$

Now, the right-hand term of Eq. (5) needs to be expanded in detail. The total differential of $\boldsymbol{\varepsilon}(p, q, x, t)$ is, 


$$
\mathrm{d} \boldsymbol{\varepsilon}(p, q, x, t)=\frac{\partial \boldsymbol{\varepsilon}}{\partial p} \mathrm{~d} p+\frac{\partial \boldsymbol{\varepsilon}}{\partial q} \mathrm{~d} q+\frac{\partial \boldsymbol{\varepsilon}}{\partial x} \mathrm{~d} x+\frac{\partial \boldsymbol{\varepsilon}}{\partial t} \mathrm{~d} t
$$

Thus, the right-hand term of Eq. (5) can be rewritten as,

$$
\mathrm{d} \mathbf{z} \wedge \mathrm{d} \boldsymbol{\varepsilon}(p, q, x, t)=\mathrm{d} \mathbf{z} \wedge \frac{\partial \boldsymbol{\varepsilon}}{\partial p} \mathrm{~d} p+\mathrm{d} \mathbf{z} \wedge \frac{\partial \boldsymbol{\varepsilon}}{\partial q} \mathrm{~d} q+\mathrm{d} \mathbf{z} \wedge \frac{\partial \boldsymbol{\varepsilon}}{\partial x} \mathrm{~d} x+\mathrm{d} \mathbf{z} \wedge \frac{\partial \boldsymbol{\varepsilon}}{\partial t} \mathrm{~d} t
$$

where, $\wedge$ is exterior product operator, and

$$
\begin{aligned}
& \frac{\partial \boldsymbol{\varepsilon}}{\partial p}=\left[-\eta \cos \left(k_{0} x-\omega_{0} t\right), \gamma-\eta \sin \left(k_{0} x-\omega_{0} t\right), 0,0\right]^{\mathrm{T}} \\
& \frac{\partial \boldsymbol{\varepsilon}}{\partial q}=\left[-\gamma+\eta \sin \left(k_{0} x-\omega_{0} t\right),-\eta \cos \left(k_{0} x-\omega_{0} t\right), 0,0\right]^{\mathrm{T}}
\end{aligned}
$$

$\frac{\partial \boldsymbol{\varepsilon}}{\partial x}=\left[-\gamma w-\eta v \cos \left(k_{0} x-\omega_{0} t\right)+\eta w \sin \left(k_{0} x-\omega_{0} t\right)+k_{0} \eta q \cos \left(k_{0} x-\omega_{0} t\right)+k_{0} \eta p \sin \left(k_{0} x-\omega_{0} t\right)\right.$,

$\left.\gamma v-\eta v \sin \left(k_{0} x-\omega_{0} t\right)-\eta w \cos \left(k_{0} x-\omega_{0} t\right)+k_{0} \eta q \sin \left(k_{0} x-\omega_{0} t\right)-k_{0} \eta p \cos \left(k_{0} x-\omega_{0} t\right), 0,0\right]^{\mathrm{T}}$

$$
\begin{aligned}
& \frac{\partial \boldsymbol{\varepsilon}}{\partial t}=\left[-\gamma \partial_{t} q-\eta \partial_{t} p \cos \left(k_{0} x-\omega_{0} t\right)+\eta \partial_{t} q \sin \left(k_{0} x-\omega_{0} t\right)-\omega_{0} \eta q \cos \left(k_{0} x-\omega_{0} t\right)-\omega_{0} \eta p \sin \left(k_{0} x-\omega_{0} t\right),\right. \\
& \left.\gamma \partial_{t} p-\eta \partial_{t} p \sin \left(k_{0} x-\omega_{0} t\right)-\eta \partial_{t} q \cos \left(k_{0} x-\omega_{0} t\right)-\omega_{0} \eta q \sin \left(k_{0} x-\omega_{0} t\right)+\omega_{0} \eta p \cos \left(k_{0} x-\omega_{0} t\right), 0,0\right]^{\mathrm{T}}
\end{aligned}
$$

Then, the exterior product operations contained in Eq. (7) can be performed as,

$$
\begin{gathered}
\mathrm{d} \mathbf{z} \wedge \frac{\partial \boldsymbol{\varepsilon}}{\partial p} \mathrm{~d} p=-\eta \cos \left(k_{0} x-\omega_{0} t\right) \mathrm{d} p \wedge \mathrm{d} p-\left[-\gamma+\eta \sin \left(k_{0} x-\omega_{0} t\right)\right] \mathrm{d} q \wedge \mathrm{d} p \\
=\left[-\gamma+\eta \sin \left(k_{0} x-\omega_{0} t\right)\right] \mathrm{d} p \wedge \mathrm{d} q \\
\mathrm{~d} \mathbf{z} \wedge \frac{\partial \boldsymbol{\varepsilon}}{\partial q} \mathrm{~d} q=\left[-\gamma+\eta \sin \left(k_{0} x-\omega_{0} t\right)\right] \mathrm{d} p \wedge \mathrm{d} q-\eta \cos \left(k_{0} x-\omega_{0} t\right) \mathrm{d} q \wedge \mathrm{d} q \\
=\left[-\gamma+\eta \sin \left(k_{0} x-\omega_{0} t\right)\right] \mathrm{d} p \wedge \mathrm{d} q \\
\mathrm{~d} \mathbf{z} \wedge \frac{\partial \boldsymbol{\varepsilon}}{\partial x} \mathrm{~d} x=\left[-\gamma w-\eta v \cos \left(k_{0} x-\omega_{0} t\right)+\eta w \sin \left(k_{0} x-\omega_{0} t\right)+k_{0} \eta q \cos \left(k_{0} x-\omega_{0} t\right)\right. \\
\left.+k_{0} \eta p \sin \left(k_{0} x-\omega_{0} t\right)\right] \mathrm{d} p \wedge \mathrm{d} x+\left[\gamma v-\eta v \sin \left(k_{0} x-\omega_{0} t\right)-\eta w \cos \left(k_{0} x-\omega_{0} t\right)+\right. \\
\left.k_{0} \eta q \sin \left(k_{0} x-\omega_{0} t\right)-k_{0} \eta p \cos \left(k_{0} x-\omega_{0} t\right)\right] \mathrm{d} q \wedge \mathrm{d} x \\
=\gamma[v \mathrm{~d} q \wedge \mathrm{d} x-w \mathrm{~d} p \wedge \mathrm{d} x]+\eta\left\{\left[v \cos \left(k_{0} x-\omega_{0} t\right)+w \sin \left(k_{0} x-\omega_{0} t\right)+\right.\right. \\
\left.k_{0} q \cos \left(k_{0} x-\omega_{0} t\right)+k_{0} p \sin \left(k_{0} x-\omega_{0} t\right)\right] \mathrm{d} p \wedge \mathrm{d} x+\left[-v \sin \left(k_{0} x-\omega_{0} t\right)-w \cos \left(k_{0} x-\right.\right. \\
\left.\left.\left.\omega_{0} t\right)+k_{0} q \sin \left(k_{0} x-\omega_{0} t\right)-k_{0} p \cos \left(k_{0} x-\omega_{0} t\right)\right] \mathrm{d} q \wedge \mathrm{d} x\right\}
\end{gathered}
$$




$$
\begin{aligned}
& \quad \mathrm{d} \mathbf{z} \wedge \frac{\partial \boldsymbol{\varepsilon}}{\partial t} \mathrm{~d} t=\left[-\gamma \partial_{t} q-\eta \partial_{t} p \cos \left(k_{0} x-\omega_{0} t\right)+\eta \partial_{t} q \sin \left(k_{0} x-\omega_{0} t\right)-\omega_{0} \eta q \cos \left(k_{0} x-\omega_{0} t\right)\right. \\
& \left.-\omega_{0} \eta p \sin \left(k_{0} x-\omega_{0} t\right)\right] \mathrm{d} p \wedge \mathrm{d} t+\left[-\gamma \partial_{t} p-\eta \partial_{t} p \sin \left(k_{0} x-\omega_{0} t\right)-\eta \partial_{t} q \cos \left(k_{0} x-\omega_{0} t\right)-\right. \\
& \begin{array}{c}
\left.\omega_{0} \eta q \sin \left(k_{0} x-\omega_{0} t\right)+\omega_{0} \eta p \cos \left(k_{0} x-\omega_{0} t\right)\right] \mathrm{d} q \wedge \mathrm{d} t \\
\quad=\gamma\left[\partial_{t} p \mathrm{~d} q \wedge \mathrm{d} t-\partial_{t} q \mathrm{~d} p \wedge \mathrm{d} t\right]+\eta\left\{\left[-\partial_{t} p \cos \left(k_{0} x-\omega_{0} t\right)+\partial_{t} q \sin \left(k_{0} x-\omega_{0} t\right)\right.\right. \\
\left.-\omega_{0} q \cos \left(k_{0} x-\omega_{0} t\right)-\omega_{0} p \sin \left(k_{0} x-\omega_{0} t\right)\right] \mathrm{d} p \wedge \mathrm{d} t+\left[-\partial_{t} p \sin \left(k_{0} x-\omega_{0} t\right)-\partial_{t} q \cos \left(k_{0} x-\right.\right. \\
\left.\left.\left.\omega_{0} t\right)-\omega_{0} q \sin \left(k_{0} x-\omega_{0} t\right)+\omega_{0} p \cos \left(k_{0} x-\omega_{0} t\right)\right] \mathrm{d} q \wedge \mathrm{d} t\right\}
\end{array}
\end{aligned}
$$

Substituting Eqs. (8)-(11) into Eq. (7), the expanding form of the right-hand term of Eq. (5) can be obtained,

$$
\begin{aligned}
& \Delta=\mathrm{d} \mathbf{z} \wedge \mathrm{d} \boldsymbol{\varepsilon}(p, q, x, t)=-\gamma\left[2 \mathrm{~d} p \wedge \mathrm{d} q+w \mathrm{~d} p \wedge \mathrm{d} x-v \mathrm{~d} q \wedge \mathrm{d} x+\partial_{t} q \mathrm{~d} p \wedge \mathrm{d} t-\partial_{t} p \mathrm{~d} q \wedge \mathrm{d} t\right]+ \\
& \eta\left\{2 \sin \left(k_{0} x-\omega_{0} t\right) \mathrm{d} p \wedge \mathrm{d} q+\left[v \cos \left(k_{0} x-\omega_{0} t\right)+w \sin \left(k_{0} x-\omega_{0} t\right)+k_{0} q \cos \left(k_{0} x-\omega_{0} t\right)\right.\right. \\
& \left.+k_{0} p \sin \left(k_{0} x-\omega_{0} t\right)\right] \mathrm{d} p \wedge \mathrm{d} x+\left[-v \sin \left(k_{0} x-\omega_{0} t\right)-w \cos \left(k_{0} x-\omega_{0} t\right)+k_{0} q \sin \left(k_{0} x-\omega_{0} t\right)\right. \\
& \left.-k_{0} p \cos \left(k_{0} x-\omega_{0} t\right)\right] \mathrm{d} q \wedge \mathrm{d} x+\left[-\partial_{t} p \cos \left(k_{0} x-\omega_{0} t\right)+\partial_{t} q \sin \left(k_{0} x-\omega_{0} t\right)-\omega_{0} q \cos \left(k_{0} x-\right.\right. \\
& \left.\left.\omega_{0} t\right)-\omega_{0} p \sin \left(k_{0} x-\omega_{0} t\right)\right] \mathrm{d} p \wedge \mathrm{d} t+\left[-\partial_{t} p \sin \left(k_{0} x-\omega_{0} t\right)-\partial_{t} q \cos \left(k_{0} x-\omega_{0} t\right)-\omega_{0} q\right. \\
& \left.\left.\sin \left(k_{0} x-\omega_{0} t\right)+\omega_{0} p \cos \left(k_{0} x-\omega_{0} t\right)\right] \mathrm{d} q \wedge \mathrm{d} t\right\}
\end{aligned}
$$

where $\Delta$ is named as the generalized multi-symplectic conservation law perturbation $[43,45]$.

Form Eq. (12), it can be found that the form of the generalized multi-symplectic conservation law perturbation is similar to those presented in our previous jobs $[43,45]$ even if the damping rate is not contained in the coefficient matrices of Eq. (4), but in $\boldsymbol{\varepsilon}(p, q, x, t)$. In this paper, the focus of the numerical method is not on the generalized multi-symplectic conservation law perturbation but on the energy loss as well as the momentum loss instead.

It is well known that the linear damping and the external perturbation may break the conservation law of the local energy $[44,46]$, which can be illustrated as follows. Taking the inner product of Eq. (4) with $\partial_{t} \mathbf{z}$, for the skew-symmetry of $\mathbf{M}$ that implies $\left\langle\partial_{t} \mathbf{z}, \mathbf{M} \partial_{t} \mathbf{z}\right\rangle=0$, then we can obtain,

$$
\left\langle\partial_{t} \mathbf{z}, \mathbf{K} \partial_{x} \mathbf{z}\right\rangle=\left\langle\partial_{t} \mathbf{z}, \nabla_{\mathbf{z}} S(\mathbf{z})\right\rangle+\left\langle\partial_{t} \mathbf{z}, \mathbf{\varepsilon}(p, q, x, t)\right\rangle
$$

Noting that $\left\langle\partial_{t} \mathbf{z}, \mathbf{K} \partial_{x} \mathbf{z}\right\rangle=\frac{1}{2} \partial_{t}\left\langle\mathbf{z}, \mathbf{K} \partial_{x} \mathbf{z}\right\rangle+\frac{1}{2} \partial_{x}\left\langle\partial_{t} \mathbf{z}, \mathbf{K z}\right\rangle$ and $\left\langle\partial_{t} \mathbf{z}, \nabla_{\mathbf{z}} S(\mathbf{z})\right\rangle=\partial_{t} S(\mathbf{z})$, Eq.

(13) can be rewritten as the modified local energy conservation law form,

$$
\partial_{t}\left[S(\mathbf{z})-\frac{1}{2}\left\langle\mathbf{z}, \mathbf{K} \partial_{x} \mathbf{z}\right\rangle\right]+\frac{1}{2} \partial_{x}\left\langle\mathbf{z}, \mathbf{K} \partial_{t} \mathbf{z}\right\rangle=\Delta_{e}
$$


where, $\Delta_{e}$ is named as the perturbation of the modified local energy [43] that can be expressed as

$$
\begin{aligned}
\Delta_{e}= & \left\langle\partial_{t} \mathbf{z}, \boldsymbol{\varepsilon}(p, q, x, t)\right\rangle \\
= & \partial_{t} p\left[-\gamma q-\eta p \cos \left(k_{0} x-\omega_{0} t\right)+\eta q \sin \left(k_{0} x-\omega_{0} t\right)\right]+ \\
& \partial_{t} q\left[\gamma p-\eta p \sin \left(k_{0} x-\omega_{0} t\right)-\eta q \cos \left(k_{0} x-\omega_{0} t\right)\right]
\end{aligned}
$$

It is interesting that, in a time period of the external perturbation, the integration of $\Delta_{e}$ is,

$$
\begin{aligned}
\Delta_{e T} & =\int_{t_{0}}^{\frac{2 \pi}{\omega_{0}}+t_{0}} \Delta_{e} \mathrm{~d} t \\
& =\int_{t_{0}}^{\frac{2 \pi}{\omega_{0}}+t_{0}}\left\langle\partial_{t} \mathbf{z}, \boldsymbol{\varepsilon}(p, q, x, t)\right\rangle \mathrm{d} t \\
& =-\gamma \int_{t_{0}}^{\frac{2 \pi}{\omega_{0}}+t_{0}}\left(q \partial_{t} p-p \partial_{t} q\right) \mathrm{d} t
\end{aligned}
$$

which is independent of $\eta$ and implies that the local energy dissipation on optional position $x$ in an arbitrary time period of the external perturbation is independent of the external periodic perturbation. Where $t_{0}$ is an arbitrary moment.

Similarly, in a wavelength range of the external perturbation, the integration of $\Delta_{e}$ is,

$$
\begin{aligned}
\Delta_{e \lambda} & =\int_{x_{0}}^{\frac{2 \pi}{k_{0}}+x_{0}} \Delta_{e} \mathrm{~d} x \\
& =\int_{x_{0}}^{\frac{2 \pi}{k_{0}}+x_{0}}\left\langle\partial_{t} \mathbf{z}, \mathbf{\varepsilon}(p, q, x, t)\right\rangle \mathrm{d} x \\
& =-\gamma \int_{x_{0}}^{\frac{2 \pi}{k_{0}}+x_{0}}\left(q \partial_{t} p-p \partial_{t} q\right) \mathrm{d} x
\end{aligned}
$$

which is independent of $\eta$ and implies that the local energy dissipation at optional moment $t$ in an arbitrary wavelength range is also independent of the external periodic perturbation. Where $x_{0}$ is an arbitrary position.

Similar to the deducing process for the modified local energy conservation law mentioned above, the modified local momentum conservation law can be obtained by taking the inner product of Eq. (4) with $\partial_{x} \mathbf{z}$,

$$
\partial_{x}\left[S(\mathbf{z})-\frac{1}{2}\left\langle\mathbf{z}, \mathbf{M} \partial_{t} \mathbf{z}\right\rangle\right]+\frac{1}{2} \partial_{t}\left\langle\mathbf{z}, \mathbf{M} \partial_{x} \mathbf{z}\right\rangle=\Delta_{p}
$$

where, $\Delta_{p}$ is named as the perturbation of the modified local momentum [43] that can be expressed as 


$$
\begin{aligned}
\Delta_{p}= & \left\langle\partial_{x} \mathbf{z}, \boldsymbol{\varepsilon}(p, q, x, t)\right\rangle \\
= & \partial_{x} p\left[-\gamma q-\eta p \cos \left(k_{0} x-\omega_{0} t\right)+\eta q \sin \left(k_{0} x-\omega_{0} t\right)\right]+ \\
& \partial_{x} q\left[\gamma p-\eta p \sin \left(k_{0} x-\omega_{0} t\right)-\eta q \cos \left(k_{0} x-\omega_{0} t\right)\right]
\end{aligned}
$$

The independence between $\eta$ with the local momentum change in an arbitrary time period and in an arbitrary wavelength range can be illustrated by the following two integrations of $\Delta_{p}$,

$$
\begin{aligned}
& \Delta_{p T}=\int_{t_{0}}^{\frac{2 \pi}{\omega_{0}}+t_{0}} \Delta_{p} \mathrm{~d} t=-\gamma \int_{t_{0}}^{\frac{2 \pi}{\omega_{0}}+t_{0}}\left(q \partial_{x} p-p \partial_{x} q\right) \mathrm{d} t \\
& \Delta_{p \lambda}=\int_{x_{0}}^{\frac{2 \pi}{k_{0}}+x_{0}} \Delta_{p} \mathrm{~d} x=-\gamma \int_{x_{0}}^{\frac{2 \pi}{k_{0}}+x_{0}}\left(q \partial_{x} p-p \partial_{x} q\right) \mathrm{d} x
\end{aligned}
$$

\section{A six-point scheme for damping NLSE}

As the time-space version of the implicit midpoint rule for ordinary differential equations, the Preissmann box scheme [47] for the conservative PDEs has been proved to be multi-symplectic that could preserve the conservation laws of the local energy and the local momentum if $S$ is quadratic [48]. In this section, a six-point scheme that may own the above conservative properties of the Preissmann box scheme approximately for the NLSE (1) with small perturbation $\boldsymbol{\varepsilon}(p, q, x, t)$ will be constructed. The reasons of choosing the Preissmann box method to discrete the NLSE (1) although there are several other effective numerical methods those may own better convergence or higher accuracy mainly include: firstly, the Preissmann box scheme for conservative NLSE has been proved to be multi-symplectic with long-term numerical stability, which is agree with the structure-preserving analysis topic for the damping NLSE in this paper; secondly, the purpose of this paper is to reveal the local dynamic behaviors of the damping NLSE, including some approximate conservative properties, which implies that improving the numerical accuracy is not the only focus during the numerical discretization for the damping NLSE in this paper.

Appling the implicit midpoint rule to discretize the PDEs (4) in the time and space with the time step length $\Delta t$ and spatial step length $\Delta x$ separately, we can obtain the Preissmann box scheme in the form,

$$
\mathbf{M} \delta_{t}^{+} \mathbf{z}_{j}^{k+1 / 2}+\mathbf{K} \delta_{x}^{+} \mathbf{z}_{j+1 / 2}^{k}=\nabla_{\mathbf{z}} S\left(\mathbf{z}_{j+1 / 2}^{k+1 / 2}\right)+\boldsymbol{\varepsilon}\left(p_{j+1 / 2}^{k+1 / 2}, q_{j+1 / 2}^{k+1 / 2}, k+1 / 2, j+1 / 2\right)
$$

where $\delta_{t}^{+}$and $\delta_{x}^{+}$are the forward differences for $\partial_{t}$ and $\partial_{x}$ separately, the midpoints are 
defined as $\mathbf{z}_{j}^{k+1 / 2}=\frac{1}{2}\left(\mathbf{z}_{j}^{k+1}+\mathbf{z}_{j}^{k}\right), \mathbf{z}_{j+1 / 2}^{k}=\frac{1}{2}\left(\mathbf{z}_{j+1}^{k}+\mathbf{z}_{j}^{k}\right), \mathbf{z}_{j+1 / 2}^{k+1 / 2}=\frac{1}{2}\left(\mathbf{z}_{j+1}^{k+1 / 2}+\mathbf{z}_{j}^{k+1 / 2}\right)$ and so on.

Expanding Eq. (22), we can get,

$$
\begin{gathered}
\frac{q_{j+1}^{k+1 / 2}-q_{j}^{k+1 / 2}}{\Delta t}-\alpha \frac{v_{j+1 / 2}^{k+1}-v_{j+1 / 2}^{k}}{\Delta x}=\beta\left[\left(p_{j+1 / 2}^{k+1 / 2}\right)^{2}+\left(q_{j+1 / 2}^{k+1 / 2}\right)^{2}\right] p_{j+1 / 2}^{k+1 / 2}-\gamma q_{j+1 / 2}^{k+1 / 2}- \\
\eta\left[p_{j+1 / 2}^{k+1 / 2} \cos \left(k_{0} k-\omega_{0} j\right)-q_{j+1 / 2}^{k+1 / 2} \sin \left(k_{0} k-\omega_{0} j\right)\right] \\
-\frac{p_{j+1}^{k+1 / 2}-p_{j}^{k+1 / 2}}{\Delta t}-\alpha \frac{w_{j+1 / 2}^{k+1}-w_{j+1 / 2}^{k}}{\Delta x}=\beta\left[\left(p_{j+1 / 2}^{k+1 / 2}\right)^{2}+\left(q_{j+1 / 2}^{k+1 / 2}\right)^{2}\right] q_{j+1 / 2}^{k+1 / 2}+\gamma p_{j+1 / 2}^{k+1 / 2}- \\
\eta\left[p_{j+1 / 2}^{k+1 / 2} \sin \left(k_{0} k-\omega_{0} j\right)+q_{j+1 / 2}^{k+1 / 2} \cos \left(k_{0} k-\omega_{0} j\right)\right] \\
\alpha\left(p_{j+1 / 2}^{k+1}-p_{j+1 / 2}^{k}\right) / \Delta x=\alpha v_{j+1 / 2}^{k+1 / 2} \\
\alpha\left(q_{j+1 / 2}^{k+1}-q_{j+1 / 2}^{k}\right) / \Delta x=\alpha w_{j+1 / 2}^{k+1 / 2}
\end{gathered}
$$

To eliminate the intermediate variables $v$ and $w$, we consider the above scheme in the $(k+1)$ th layer,

$$
\begin{gathered}
\frac{q_{j+1}^{k+3 / 2}-q_{j}^{k+3 / 2}}{\Delta t}-\alpha \frac{v_{j+1 / 2}^{k+2}-v_{j+1 / 2}^{k+1}}{\Delta x}=\beta\left[\left(p_{j+1 / 2}^{k+3 / 2}\right)^{2}+\left(q_{j+1 / 2}^{k+3 / 2}\right)^{2}\right] p_{j+1 / 2}^{k+3 / 2}-\gamma q_{j+1 / 2}^{k+3 / 2}-\eta\left[p_{j+1 / 2}^{k+3 / 2}\right. \\
\left.\cos \left(k_{0} k+k_{0}-\omega_{0} j\right)-q_{j+1 / 2}^{k+3 / 2} \sin \left(k_{0} k+k_{0}-\omega_{0} j\right)\right] \\
-\frac{p_{j+1}^{k+3 / 2}-p_{j}^{k+3 / 2}}{\Delta t}-\alpha \frac{w_{j+1 / 2}^{k+2}-w_{j+1 / 2}^{k+1}}{\Delta x}=\beta\left[\left(p_{j+1 / 2}^{k+3 / 2}\right)^{2}+\left(q_{j+1 / 2}^{k+3 / 2}\right)^{2}\right] q_{j+1 / 2}^{k+3 / 2}+\gamma p_{j+1 / 2}^{k+3 / 2}-\eta\left[p_{j+1 / 2}^{k+3 / 2}\right. \\
\left.\sin \left(k_{0} k+k_{0}-\omega_{0} j\right)+q_{j+1 / 2}^{k+3 / 2} \cos \left(k_{0} k+k_{0}-\omega_{0} j\right)\right] \\
\alpha\left(p_{j+1 / 2}^{k+2}-p_{j+1 / 2}^{k+1}\right) / \Delta x=\alpha v_{j+1 / 2}^{k+3 / 2} \\
\alpha\left(q_{j+1 / 2}^{k+2}-q_{j+1 / 2}^{k+1}\right) / \Delta x=\alpha w_{j+1 / 2}^{k+3 / 2}
\end{gathered}
$$

$(23)+(27)$, we can get,

$\frac{q_{j+1}^{k+3 / 2}-q_{j}^{k+3 / 2}+q_{j+1}^{k+1 / 2}-q_{j}^{k+1 / 2}}{\Delta t}-\alpha \frac{v_{j+1 / 2}^{k+2}-v_{j+1 / 2}^{k}}{\Delta x}=\beta\left[\left(p_{j+1 / 2}^{k+3 / 2}\right)^{2}+\left(q_{j+1 / 2}^{k+3 / 2}\right)^{2}\right] p_{j+1 / 2}^{k+3 / 2}-\gamma q_{j+1 / 2}^{k+3 / 2}$

$-\eta\left[p_{j+1 / 2}^{k+3 / 2} \cos \left(k_{0} k+k_{0}-\omega_{0} j\right)-q_{j+1 / 2}^{k+3 / 2} \sin \left(k_{0} k+k_{0}-\omega_{0} j\right)\right]+\beta\left[\left(p_{j+1 / 2}^{k+1 / 2}\right)^{2}+\left(q_{j+1 / 2}^{k+1 / 2}\right)^{2}\right] p_{j+1 / 2}^{k+1 / 2}$

$-\gamma q_{j+1 / 2}^{k+1 / 2}-\eta\left[p_{j+1 / 2}^{k+1 / 2} \cos \left(k_{0} k-\omega_{0} j\right)-q_{j+1 / 2}^{k+1 / 2} \sin \left(k_{0} k-\omega_{0} j\right)\right]$

(29)-(25), we can get,

$$
2 \alpha\left(p_{j+1 / 2}^{k+2}-2 p_{j+1 / 2}^{k+1}+p_{j+1 / 2}^{k}\right) / \Delta x=\alpha\left(v_{j+1 / 2}^{k+2}-v_{j+1 / 2}^{k}\right)
$$

Substituting Eq. (32) into Eq. (31), the intermediate variable $v$ is eliminated, 


$$
\begin{aligned}
& \frac{q_{j+1}^{k+3 / 2}-q_{j}^{k+3 / 2}+q_{j+1}^{k+1 / 2}-q_{j}^{k+1 / 2}}{\Delta t}-2 \alpha \frac{p_{j+1 / 2}^{k+2}-2 p_{j+1 / 2}^{k+1}+p_{j+1 / 2}^{k}}{(\Delta x)^{2}}=\beta\left[\left(p_{j+1 / 2}^{k+3 / 2}\right)^{2}+\left(q_{j+1 / 2}^{k+3 / 2}\right)^{2}\right] \\
& p_{j+1 / 2}^{k+3 / 2}-\gamma q_{j+1 / 2}^{k+3 / 2}-\eta\left[p_{j+1 / 2}^{k+3 / 2} \cos \left(k_{0} k+k_{0}-\omega_{0} j\right)-q_{j+1 / 2}^{k+3 / 2} \sin \left(k_{0} k+k_{0}-\omega_{0} j\right)\right]+\beta\left[\left(p_{j+1 / 2}^{k+1 / 2}\right)^{2}\right. \\
& \left.+\left(q_{j+1 / 2}^{k+1 / 2}\right)^{2}\right] p_{j+1 / 2}^{k+1 / 2}-\gamma q_{j+1 / 2}^{k+1 / 2}-\eta\left[p_{j+1 / 2}^{k+1 / 2} \cos \left(k_{0} k-\omega_{0} j\right)-q_{j+1 / 2}^{k+1 / 2} \sin \left(k_{0} k-\omega_{0} j\right)\right]
\end{aligned}
$$

Similarly, the variable $w$ can be eliminated from Eqs. (24), (26),(28) and (30),

$$
\begin{aligned}
& -\frac{p_{j+1}^{k+3 / 2}-p_{j}^{k+3 / 2}+p_{j+1}^{k+1 / 2}-p_{j}^{k+1 / 2}}{\Delta t}-2 \alpha \frac{q_{j+1 / 2}^{k+2}-2 q_{j+1 / 2}^{k+1}+q_{j+1 / 2}^{k}}{(\Delta x)^{2}}=\beta\left[\left(p_{j+1 / 2}^{k+3 / 2}\right)^{2}+\left(q_{j+1 / 2}^{k+3 / 2}\right)^{2}\right] \\
& q_{j+1 / 2}^{k+3 / 2}+\gamma p_{j+1 / 2}^{k+3 / 2}-\eta\left[p_{j+1 / 2}^{k+3 / 2} \sin \left(k_{0} k+k_{0}-\omega_{0} j\right)+q_{j+1 / 2}^{k+3 / 2} \cos \left(k_{0} k+k_{0}-\omega_{0} j\right)\right]+\beta\left[\left(p_{j+1 / 2}^{k+1 / 2}\right)^{2}\right. \\
& \left.+\left(q_{j+1 / 2}^{k+1 / 2}\right)^{2}\right] q_{j+1 / 2}^{k+1 / 2}+\gamma p_{j+1 / 2}^{k+1 / 2}-\eta\left[p_{j+1 / 2}^{k+1 / 2} \sin \left(k_{0} k-\omega_{0} j\right)+q_{j+1 / 2}^{k+1 / 2} \cos \left(k_{0} k-\omega_{0} j\right)\right]
\end{aligned}
$$

Combining Eq. (33) with Eq. (34), a six-point scheme equivalent to the Preissmann box scheme is obtained in the form,

$$
\begin{aligned}
& i \frac{u_{j+1}^{k+3 / 2}+u_{j+1}^{k+1 / 2}-u_{j}^{k+3 / 2}-p_{j}^{k+1 / 2}}{\Delta t}+2 \alpha \frac{u_{j+1 / 2}^{k+2}-2 u_{j+1 / 2}^{k+1}+u_{j+1 / 2}^{k}}{(\Delta x)^{2}}=-\beta\left(u_{j+1 / 2}^{k+3 / 2}\right)^{2}\left(u_{j+1 / 2}^{k+1 / 2}+u_{j+1 / 2}^{k+3 / 2}\right) \\
& -\gamma\left(u_{j+1 / 2}^{k+1 / 2}+u_{j+1 / 2}^{k+3 / 2}\right)+\eta\left[u_{j+1 / 2}^{k+3 / 2} \sin \left(k_{0} k+k_{0}-\omega_{0} j\right)+u_{j+1 / 2}^{k+3 / 2} \cos \left(k_{0} k+k_{0}-\omega_{0} j\right)\right]+\beta\left[\left(u_{j+1 / 2}^{k+1 / 2}\right)^{2}\right. \\
& \left.+\left(u_{j+1 / 2}^{k+1 / 2}\right)^{2}\right] u_{j+1 / 2}^{k+1 / 2}+\eta\left[u_{j+1 / 2}^{k+1 / 2} \sin \left(k_{0} k-\omega_{0} j\right)+u_{j+1 / 2}^{k+1 / 2} \cos \left(k_{0} k-\omega_{0} j\right)\right]
\end{aligned}
$$

But scheme (35) isn't convenient to be used in the numerical experiments because $u$ is a complex function, thus, the coupled schemes (33) and (34), which are equivalent to scheme (35), will be used in the numerical experiments instead.

To ensure the structure-preserving properties of this six-point scheme, the discretized generalized multi-symplectic conservation law perturbation should be no more than the truncation perturbation of difference scheme [43], that is,

$$
(\Delta)_{j}^{k} \leq O(\Delta t)+O(\Delta x)^{2}
$$

where, the discretized generalized multi-symplectic conservation law perturbation is,

$$
\begin{aligned}
(\Delta)_{j}^{k}= & -\gamma\left[2 \mathrm{~d} p_{j+1 / 2}^{k+1 / 2} \wedge \mathrm{d} q_{j+1 / 2}^{k+1 / 2}+w_{j+1 / 2}^{k+1 / 2} \mathrm{~d} p_{j+1 / 2}^{k+1 / 2} \wedge \mathrm{d} x_{j+1 / 2}^{k+1 / 2}-v_{j+1 / 2}^{k+1 / 2} \mathrm{~d} q_{j+1 / 2}^{k+1 / 2} \wedge \Delta x+\delta_{t}^{+} q_{j}^{k+1 / 2} \mathrm{~d} p_{j+1 / 2}^{k+1 / 2} \wedge \Delta t-\right. \\
& \left.\delta_{t}^{+} p_{j}^{k+1 / 2} \mathrm{~d} q_{j+1 / 2}^{k+1 / 2} \wedge \Delta t\right]+\eta\left\{2 \sin \left(k_{0} k-\omega_{0} j\right) \mathrm{d} p_{j+1 / 2}^{k+1 / 2} \wedge \mathrm{d} q_{j+1 / 2}^{k+1 / 2}+\left[v_{j+1 / 2}^{k+1 / 2} \cos \left(k_{0} k-\omega_{0} j\right)+w_{j+1 / 2}^{k+1 / 2}\right.\right. \\
& \left.\sin \left(k_{0} k-\omega_{0} j\right)+k_{0} q_{j+1 / 2}^{k+1 / 2} \cos \left(k_{0} k-\omega_{0} j\right)+k_{0} p_{j+1 / 2}^{k+1 / 2} \sin \left(k_{0} k-\omega_{0} j\right)\right] \mathrm{d} p_{j+1 / 2}^{k+1 / 2} \wedge \Delta x+\left[-v_{j+1 / 2}^{k+1 / 2}\right. \\
& \left.\sin \left(k_{0} k-\omega_{0} j\right)-w_{j+1 / 2}^{k+1 / 2} \cos \left(k_{0} k-\omega_{0} j\right)+k_{0} q_{j+1 / 2}^{k+1 / 2} \sin \left(k_{0} k-\omega_{0} j\right)-k_{0} p_{j+1 / 2}^{k+1 / 2} \cos \left(k_{0} k-\omega_{0} j\right)\right] \\
& \mathrm{d} q_{j+1 / 2}^{k+1 / 2} \wedge \Delta x+\left[-\delta_{t}^{+} p_{j}^{k+1 / 2} \cos \left(k_{0} k-\omega_{0} j\right)+\delta_{t}^{+} q_{j}^{k+1 / 2} \sin \left(k_{0} k-\omega_{0} j\right)-\omega_{0} q_{j+1 / 2}^{k+1 / 2} \cos \left(k_{0} k-\omega_{0} j\right)\right. \\
& \left.-\omega_{0} p_{j+1 / 2}^{k+1 / 2} \sin \left(k_{0} k-\omega_{0} j\right)\right] \mathrm{d} p_{j+1 / 2}^{k+1 / 2} \wedge \Delta t+\left[-\delta_{t}^{+} p_{j}^{k+1 / 2} \sin \left(k_{0} k-\omega_{0} j\right)-\delta_{t}^{+} q_{j}^{k+1 / 2} \cos \left(k_{0} k-\omega_{0} j\right)\right. \\
& \left.\left.-\omega_{0} q_{j+1 / 2}^{k+1 / 2} \sin \left(k_{0} k-\omega_{0} j\right)+\omega_{0} p_{j+1 / 2}^{k+1 / 2} \cos \left(k_{0} k-\omega_{0} j\right)\right] \mathrm{d} q_{j+1 / 2}^{k+1 / 2} \wedge \Delta t\right\}
\end{aligned}
$$

The corresponding discretized local energy conservation law perturbation of the scheme is, 


$$
\begin{aligned}
\left(\Delta_{e}\right)_{j}^{k}= & \delta_{t}^{+} p_{j}^{k+1 / 2}\left[-\gamma q_{j+1 / 2}^{k+1 / 2}-\eta p_{j+1 / 2}^{k+1 / 2} \cos \left(k_{0} k-\omega_{0} j\right)+\eta q_{j+1 / 2}^{k+1 / 2} \sin \left(k_{0} k-\omega_{0} j\right)\right]+ \\
& \delta_{t}^{+} q_{j}^{k+1 / 2}\left[\gamma p_{j+1 / 2}^{k+1 / 2}-\eta p_{j+1 / 2}^{k+1 / 2} \sin \left(k_{0} k-\omega_{0} j\right)-\eta q_{j+1 / 2}^{k+1 / 2} \cos \left(k_{0} k-\omega_{0} j\right)\right]
\end{aligned}
$$

and the discretized local momentum conservation law perturbation of the scheme is,

$$
\begin{aligned}
\left(\Delta_{p}\right)_{j}^{k}= & \delta_{x}^{+} p_{j+1 / 2}^{k}\left[-\gamma q_{j+1 / 2}^{k+1 / 2}-\eta p_{j+1 / 2}^{k+1 / 2} \cos \left(k_{0} k-\omega_{0} j\right)+\eta q_{j+1 / 2}^{k+1 / 2} \sin \left(k_{0} k-\omega_{0} j\right)\right]+ \\
& \delta_{x}^{+} q_{j+1 / 2}^{k}\left[\gamma p_{j+1 / 2}^{k+1 / 2}-\eta p_{j+1 / 2}^{k+1 / 2} \sin \left(k_{0} k-\omega_{0} j\right)-\eta q_{j+1 / 2}^{k+1 / 2} \cos \left(k_{0} k-\omega_{0} j\right)\right]
\end{aligned}
$$

With the local energy/momentum conservation law perturbations obtained by Eqs. (37) and (38) respectively, the numerical integrations (16), (17), (20) and (21) can be performed to test the structure-preserving properties of the differential scheme constructed.

\section{Numerical experiments}

In this section, the structure-preserving properties of the coupled schemes (33) and (34) will be tested by recording the energy loss and the momentum loss in the simulation process firstly. Then, an important physical property of the damping NLSE, the breakup of a multisoliton state [20], will be investigated by coupled schemes (33) and (34) in detail.

In the following numerical experiments, the dispersion coefficient and the coefficient of the nonlinear term are assumed as $\alpha=1, \beta=2$. To assess the structure-preserving properties of the scheme, let the time step size $\Delta t=0.05$ and the spatial step size $\Delta x=0.1$ in the domain $x \times t=[-30,30] \times[0,60]$.

\subsection{Energy loss and momentum loss}

The most direct effect resulting from the linear damping in the system (1) is the losses of energy and momentum, which have been presented analytically in Section 2, see Eqs. (15) -(17) and Eqs. (19)-(21), and will be verified numerically in this section.

In this section, the numerical experiments are preformed with the following initial condition

$$
u(x, 0)=\operatorname{sech}(x) \exp (2 i x)
$$

and the boundary conditions,

$$
\begin{aligned}
& u(-30, t)=u(30, t)=0, \quad \text { for } t \succ 0 \\
& \partial_{x} u(-30, t)=\partial_{x} u(30, t)=0, \quad \text { for } t \succ 0
\end{aligned}
$$

Referring to the theory of the generalized multi-symplectic integration [43], inequation (36) should be satisfied firstly to insure the structure-preserving properties of the schemes. 
Theoretically, the numerical results obtained by the schemes (33) and (34) will oscillate more seriously with the increase of the amplitude of $h(x, t)$. To obtain the maximum value of the linear damping coefficient, we let $\eta=0.1, k_{0}=1$ and $\omega_{0}=2$. Increasing the value of the linear damping coefficient $\gamma$ from $\gamma=0.001$ with the step length $\Delta \gamma=0.001$, inequation (36) is checked and the maximum value of the linear damping coefficient that satisfies inequation (36) narrowly is obtained. The maximum value of the linear damping coefficient is $\gamma_{\max }=0.238$ and all of the following experiments will be preformed with the linear damping coefficient no more than this threshold.

To attest the theoretical results shown in Eqs. (15)-(17) and Eqs. (19)-(21), we fix $\gamma=0.2, k_{0}=1, \omega_{0}=2$, and preform the simulation with $\eta=0.1$ and $\eta=0.01$ respectively. In the process of the simulation, the perturbation of the modified local energy and the perturbation of the modified local momentum on each grid $\left(\left(\Delta_{e}\right)_{j}^{k}\right.$ and $\left.\left(\Delta_{p}\right)_{j}^{k}\right)$ are recorded. Then, the numerical integrations are preformed for the above perturbations with respect to $x$ and $t$ respectively. The above numerical integration results $\left(\left(\Delta_{e}\right)_{j},\left(\Delta_{e}\right)^{k},\left(\Delta_{p}\right)_{j}\right.$ and $\left.\left(\Delta_{p}\right)^{k}\right)$ are shown in Figs 1 to 4. Finally, to verify the fact described by Eqs. (16), (17), (20) and (21), the numerical integrations for $\left(\Delta_{e}\right)_{j}^{k}$ and $\left(\Delta_{p}\right)_{j}^{k}$ are performed on the positions ( $x=-15(k=151)$ and $x=15(k=451))$ with respect to $t$ in each time period $\left(T=2 \pi / \omega_{0}=\pi\right.$, there are 19 integral periods from $t=0$ to $\left.t=60\right)$ and at the moments ( $t=20(j=401)$ and $t=40(j=801))$ with respect to $x$ in each wavelength range ( $\lambda=2 \pi / k_{0}=2 \pi$, there are 9 integral wavelengths from $x=-30$ to $x=30$ ). The relative deviations between the integration results of $\eta=0.01$ with those of $\eta=0.1$ at different positions/moments are plotted for each period/wavelength, see Figs. 5 and 6. From which, it can be found that the relative deviations of $\Delta_{e T}$ and $\Delta_{p T}$ are less than $10^{-11}$ for each period, and the relative deviations of $\Delta_{e \lambda}$ and $\Delta_{p \lambda}$ are less than $10^{-12}$ for each wavelength range. The tiny relative deviations presented in Figs. 5 and 6 implies that the values of $\Delta_{e T}, \Delta_{p T}, \Delta_{e \lambda}$ and $\Delta_{p \lambda}$ are independent of the amplitude of the harmonic 
excitation and the local properties described by Eqs. (16), (17), (20) and (21) are conserved by the schemes (33) and (34). In addition, the above results on $\Delta_{e T}, \Delta_{p T}, \Delta_{e \lambda}$ and $\Delta_{p \lambda}$ illustrate the tiny artificial dissipation of the numerical scheme indirectly constructed in this paper.

The numerical integration method used here to obtained $\left(\Delta_{e}\right)_{j},\left(\Delta_{e}\right)^{k},\left(\Delta_{p}\right)_{j},\left(\Delta_{p}\right)^{k}$, $\Delta_{e T}, \Delta_{p T}, \Delta_{e \lambda}$ and $\Delta_{p \lambda}$ is the 7-point Gauss-Kronrod Quadrature rule [49, 50] (The uniform formulas and the implement approach of the $(2 n+1)$-point Gauss-Kronrod Quadrature are presented in Refs. [49, 50]. Let $n=3$, the formulas of the 7-point Gauss-Kronrod Quadrature will be obtained. Thus, the explicit formulas for the numerical integrations will not be given in this paper), which has been proved to be a high-precision method for the oscillatory numerical integration that is resulted form the harmonic excitation $h(x, t)$ in our jobs.

From Figs. 1 to 4 , it can be found that the numerical results of the local energy/momentum losses respected to time/position are dependent on the amplitude of the harmonic excitation, which result from the fact that the integrating ranges $([0,60]$ respected to $t$ and $[-30,30]$ respected to $x$ ) of the local energy/momentum losses respected to time/position are not integral period/wavelength exactly. More specifically, the integrating range of the local energy/momentum losses respected to time is $60 / \pi \approx 19.099$ periods and the integrating range of the local energy/momentum losses respected to position is $60 / 2 \pi \approx 9.549$ wavelengths. Thus, the integration results of the local energy/momentum losses respected to time/position are dependent on $\eta$ observably.

The different conclusions about the dependence of the integration results on the perturbation amplitude obtained from Figs. 1-4 and Figs. 5 and 6 just illustrate the structure-preserving characteristics of the numerical scheme employed in this paper. When the integrating ranges are non-integer period/wavelength exactly, the energy/momentum losses respected to time/position are dependent on $\eta$ (see Figs 1-4), while the energy/momentum losses respected to time/position are almost independent on $\eta$ when the integrating ranges are one 
period/wavelength exactly (see Figs 5 and 6), which are agree with the analytical results presented in Eqs.(15)-(17), (19)-(21).

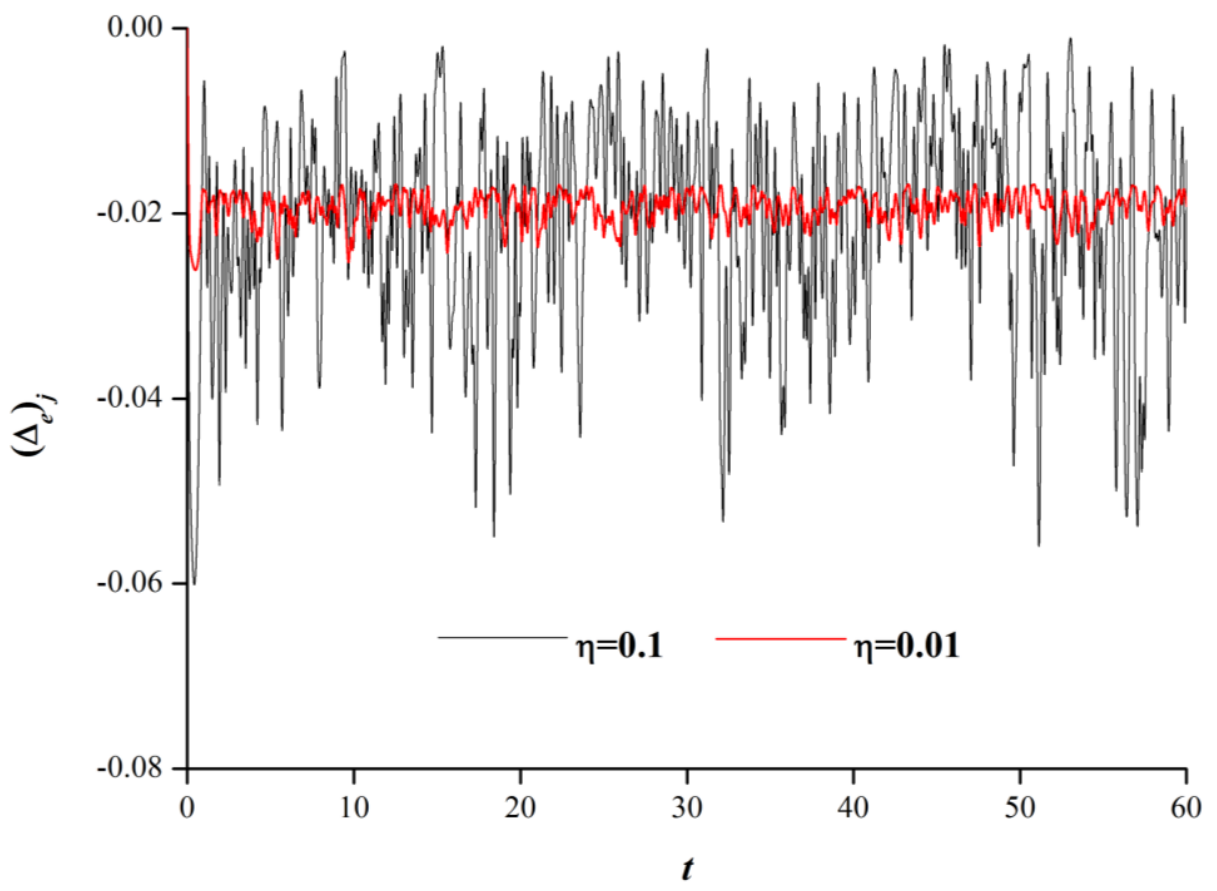

Fig. 1. Local energy loss respected to time

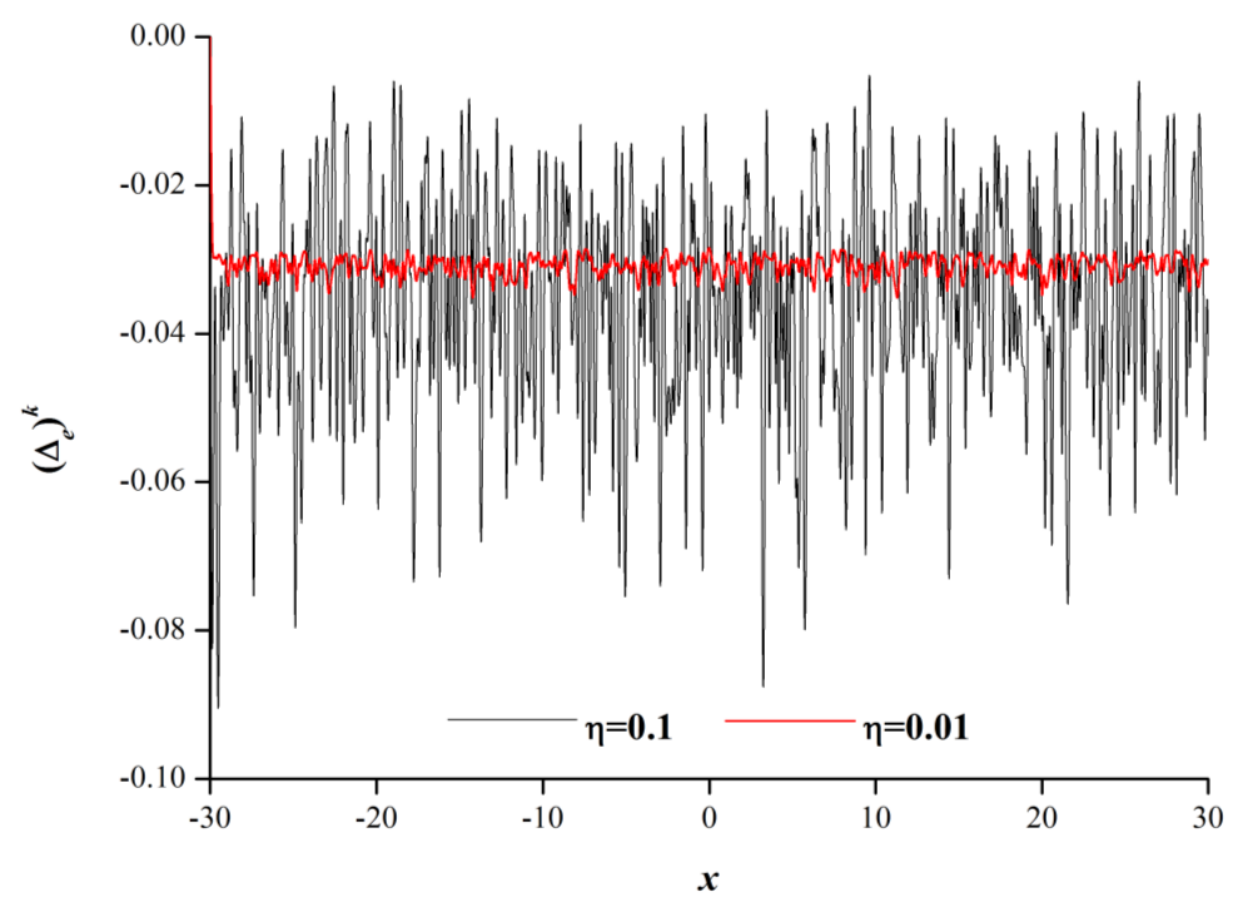

Fig. 2. Local energy loss respected to position 


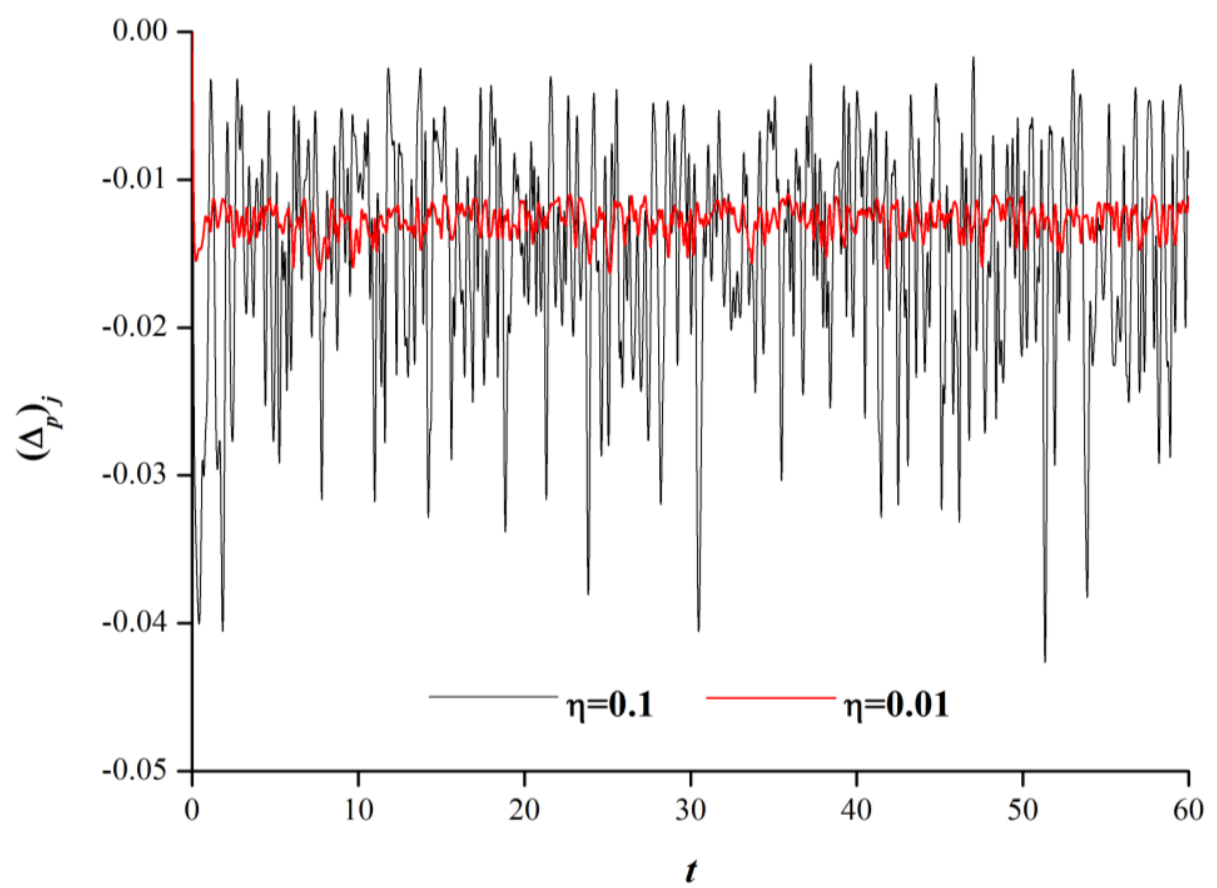

Fig. 3. Local momentum loss respected to time

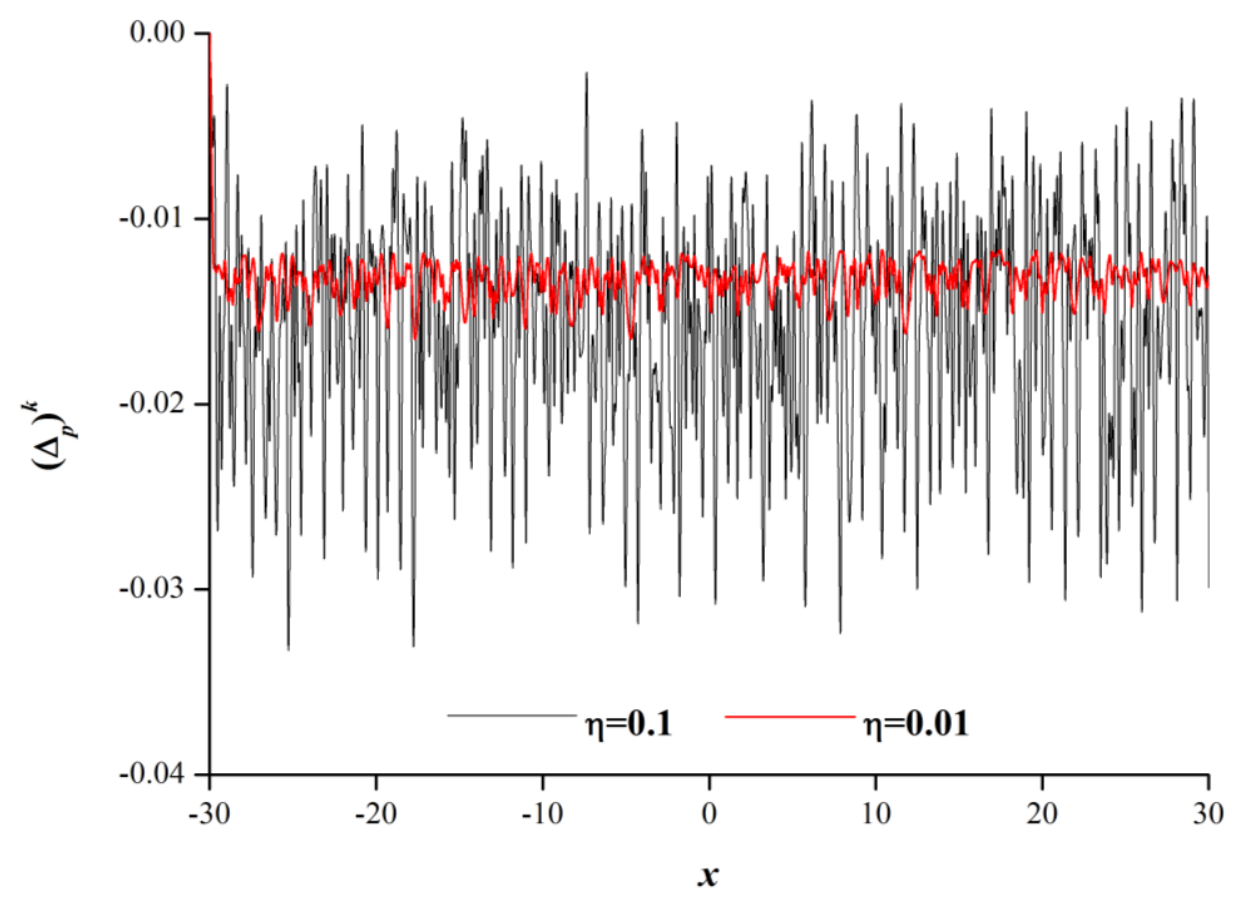

Fig. 4. Local momentum loss respected to position 


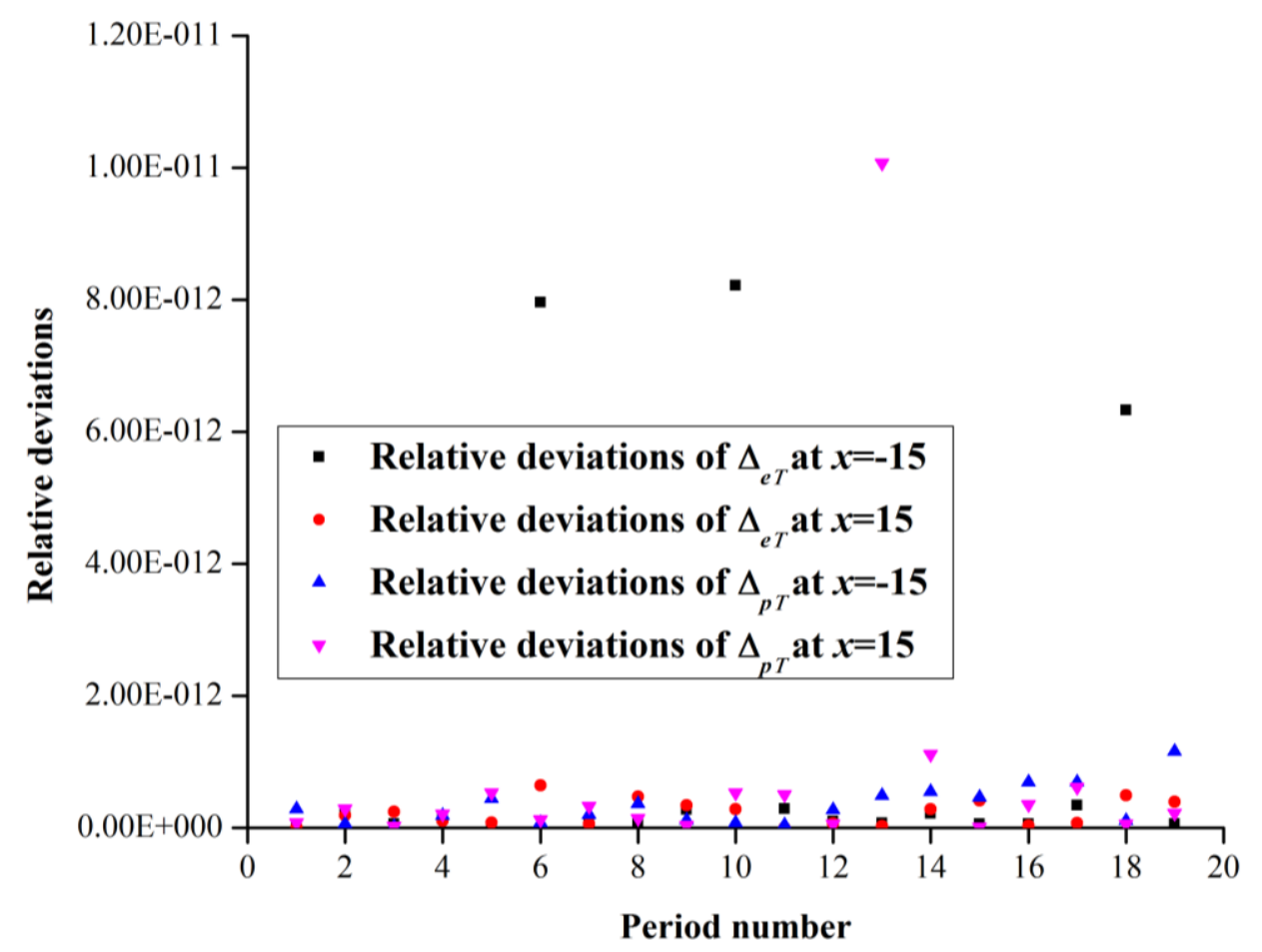

Fig. 5. Relative deviations of $\Delta_{e T}$ and $\Delta_{p T}$ for each time period

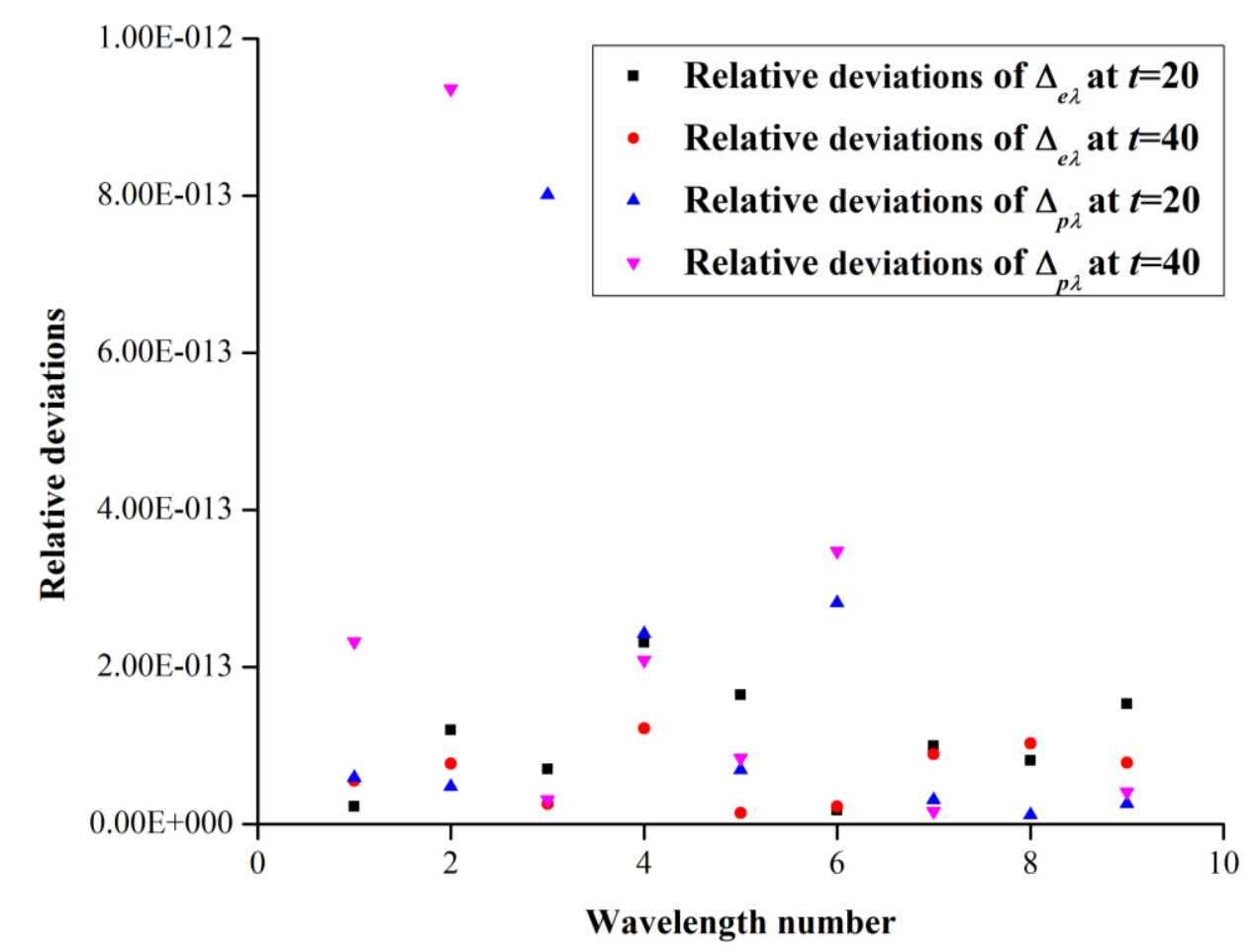

Fig. 6. Relative deviations of $\Delta_{e \lambda}$ and $\Delta_{p \lambda}$ for each wavelength range

\subsection{Breakup of multisoliton state of linearly damping NLSE}

In the previous section, the excellent structure-preserving properties on the local 
energy/momentum losses of the schemes (33) and (34) are illustrated, which implies that the schemes can be further used to investigate the local dynamic behaviors of the NLSE (1) resulting from the linearly damping and the periodic perturbation.

For the integrable NLSE without damping and perturbation, one of the most attractive local dynamic behaviors is the properties of the soliton, including the existence as well as the stability of the soliton, and so on, which have been discussed widely since the proposing of the NLSE. Here, the stability of the soliton state for the damping NLSE (1) is taken into account. It has been proved that the breather is likely to become unstable, it breaks up into individual solitons when the linearly damping is added to the integrable NLSE [20]. An important consequence of that is that whether the multisoliton state is stable or not when a periodic perturbation is added to the linearly damping NLSE, which will be investigated in this section in detail.

Let $\eta=\gamma=0.1, k_{0}=1$ and $\omega_{0}=2$, the linearly damping NLSE (1) is solved by the schemes (33) and (34) with the following initial condition, which results in the evolution of a two-soliton state for the NLSE without the damping and the perturbation,

$$
u(x, 0)=0.75 \operatorname{sech}(x-10) \exp (2 i x)+\operatorname{sech}(1.5 x+15) \exp (-2 i x)
$$

and the boundary conditions (40).

The waveforms for the real part and the imaginary part of the wave function $u$ at different moments are shown in Figs. 7 and 8 respectively. 


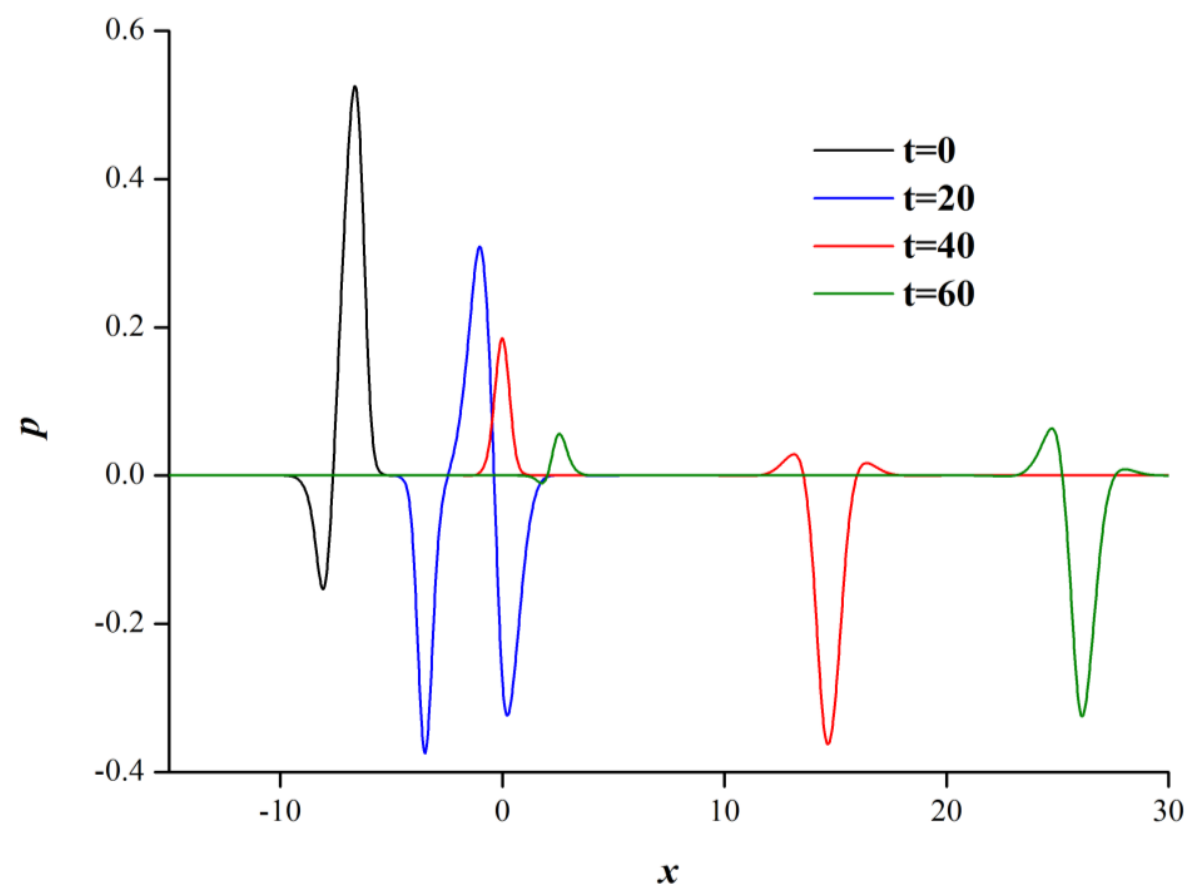

Fig. 7. Real part of the wave function

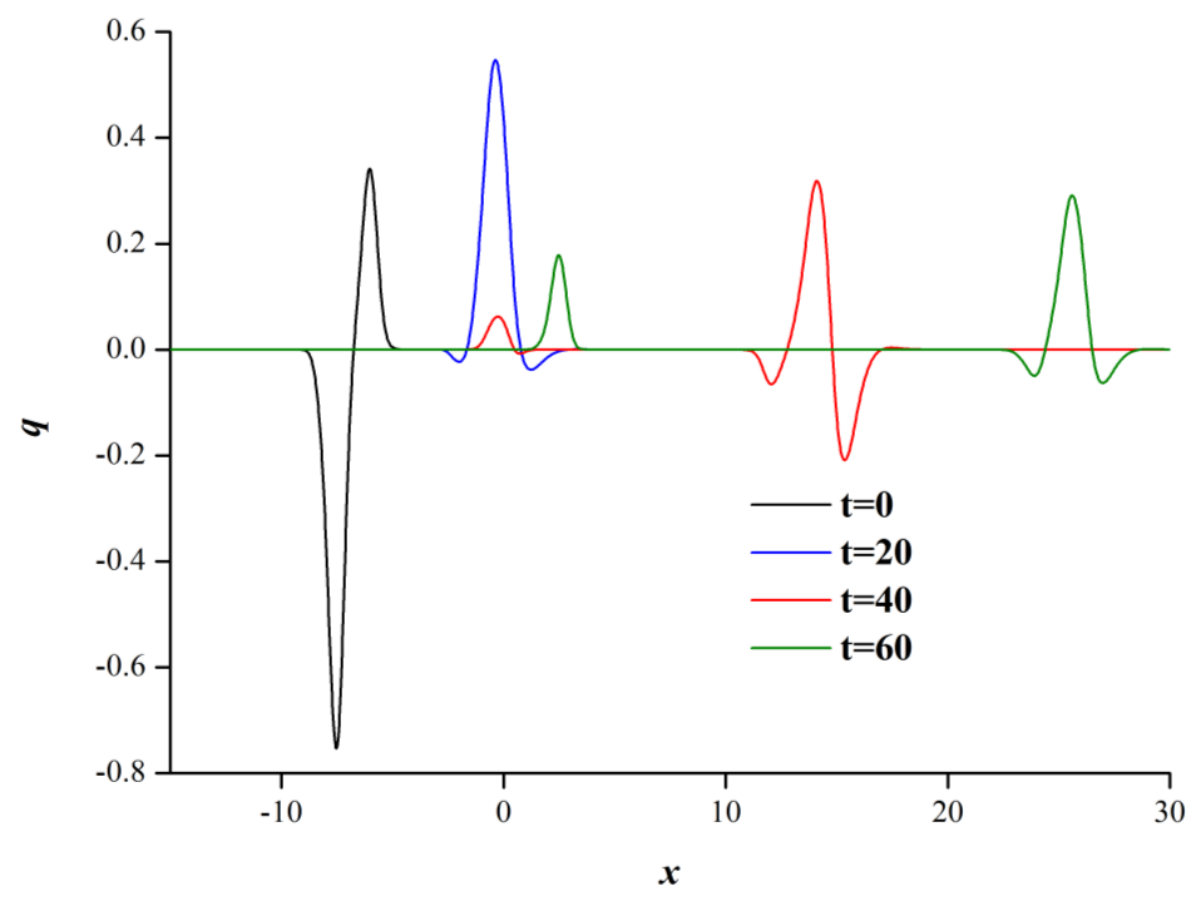

Fig. 8. Imaginary part of the wave function

In Figs. 7 and 8, the two-soliton state breaks up into three-soliton state in the time interval $0 \prec t \prec 20$. And then the three-soliton state spits into four-soliton state in the time interval $20 \prec t \prec 40$. Finally, a new individual soliton appears in the time interval $40 \prec t \prec 60$.

In those breakup processes, one can see that the amplitudes and the velocities of the 
solitons are time-varying: generally, the amplitudes of the solitons decrease with the time lapses which results from the existence of the linear damping; but, the velocities of some individual solitons decrease while the velocities of others increase, which implies that the kinetic energy transfers between the individual solitons occur in the breakup process.

For the unperturbed NLSE, the decomposition of the solution, including multisolitons, can be determined solely by the discrete eigenvalues of the associated Zakharov-Shabat spectral problem (ZSSP) [51]. Since the linear damping and the periodic perturbation are small in this paper, the decomposition of the multisoliton state at any moments can be determined approximately by the discrete eigenvalues of the associated ZSSP for the unperturbed NLSE. Thus, to investigate the dynamic behaviors in the above breakup process of the multisoliton state in detail, the discrete eigenvalues of the associated ZSSP are further considered.

With small linearly damping and small periodic perturbation, the NLSE (1) is the compatibility condition of the following linear equations approximately [20,51],

$$
\left[\begin{array}{cc}
-\partial_{x} & u \\
\bar{u} & \partial_{x}
\end{array}\right] \mathbf{Y}=i \zeta \mathbf{Y}
$$

where $\zeta=\vartheta+i \xi$ is the complex eigenvalue of the associated ZSSP, $\mathbf{Y}=\left[y_{1}, y_{2}\right]^{\mathrm{T}}, \bar{u}$ is the complex conjugation of the wave function $u$.

It is well known that the basic dynamic behaviors of the multisoliton state can be depicted by the variation of the complex eigenvalue $\zeta$ [51]: each discrete eigenvalue $\zeta_{n}=\vartheta_{n}+i \xi_{n}$ corresponds to a unique individual soliton (the real part $\vartheta_{n}$ determining the velocity of the soliton and the imaginary part $\xi_{n}$ determining the amplitude of the soliton). Thus, after the simulation on the damping NLSE (1) by the almost structure-preserving schemes (33) and (34) under the initial condition (41) and the boundary conditions (40), we can substitute the numerical result of the complex wave function on each grid $\left(u_{j}^{k}\right)$ into Eq. (42) and solve the discrete form of the eigenvalue equation (42) to obtain each discrete eigenvalue $\zeta_{n}$. Here, the numerical approach employed to obtain each discrete eigenvalue is the Fourier collocation method that was proposed by Boyd [52] and has been proved to be a 
reliable method for the eigenvalue problems with high-accuracy.

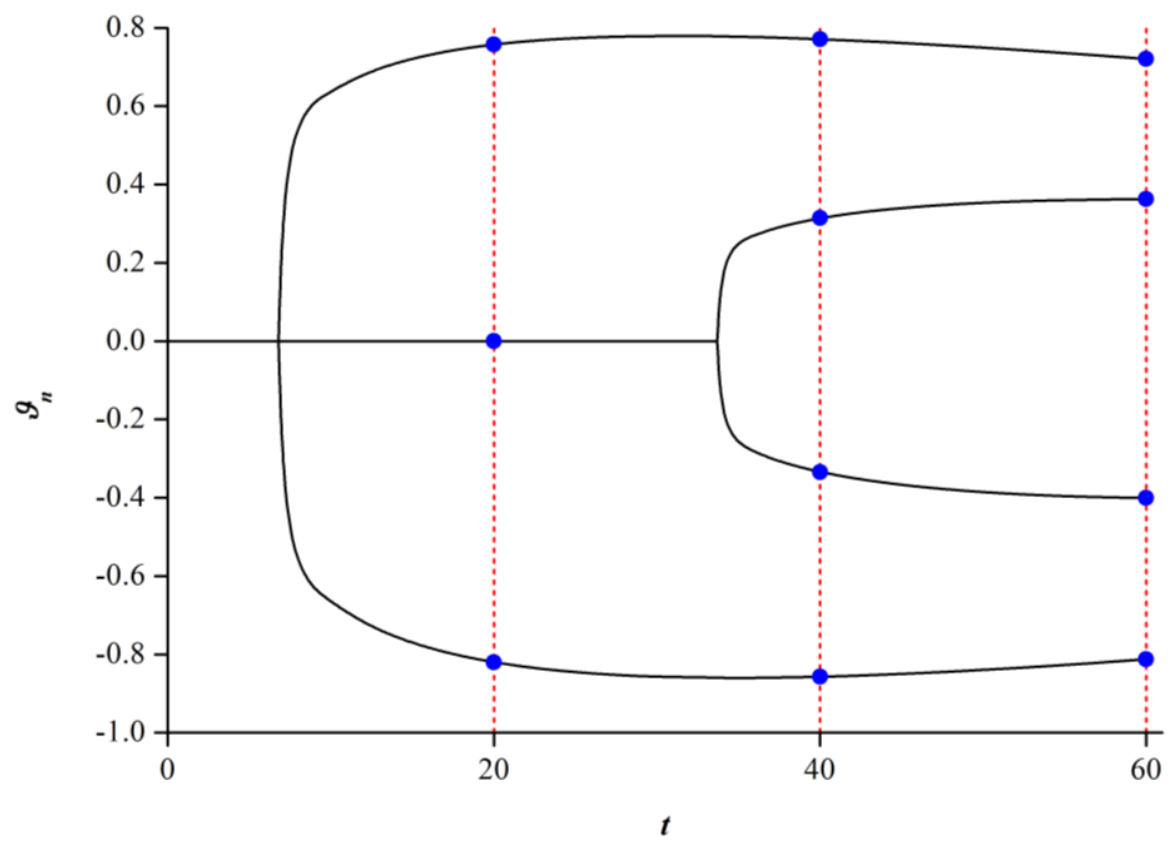

Fig. 9. Real part of eigenvalues

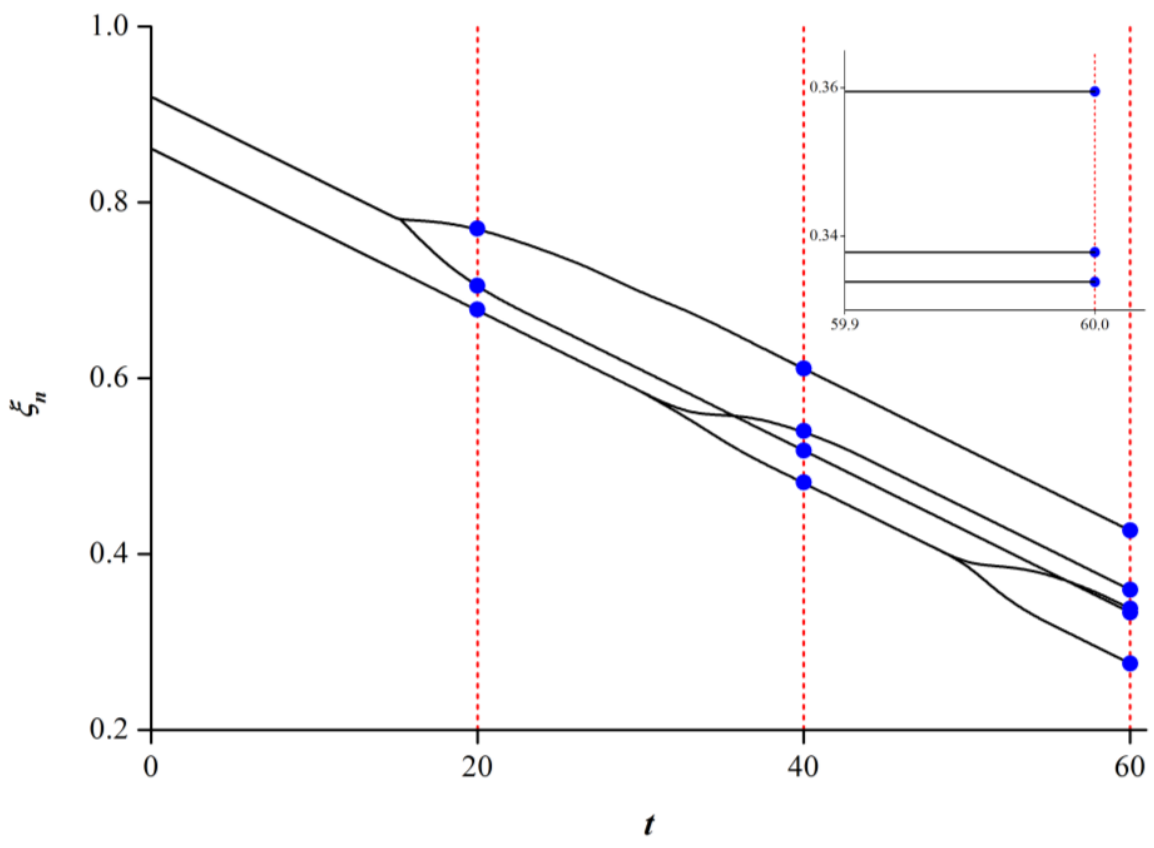

Fig. 10. Imaginary part of eigenvalues

Following the process of the Fourier collocation method proposed in Refs. [51, 52], the eigenvalue on each spatial point is obtained, which is shown in Fig. 9 and 10. In Fig. 9, two of the real part for the eigenvalues decrease and other two branches increase, which implies that the kinetic energy transfer exist between the associated solitons because the real part determines the velocity of the soliton. In Figs. 10, all branches of the imaginary part for the 
eigenvalues decrease with the time elapses, which implies that the amplitude of each soliton decrease and the loss of the potential energy for each individual soliton is verified.

Comparing the bifurcation results of eigenvalues with those presented in Ref. [20], one may conclude that: (1) Different from the evolution of the real part of the eigenvalues for the associated ZSSP presented in Ref. [20], the evolution of the real part of the eigenvalues shown in Fig. 9 is not symmetric strictly, which is resulted from the introducing the periodic perturbation term in the NLSE (1); (2) As for the evolution of the imaginary part of the eigenvalues for the associated ZSSP, the imaginary part of the eigenvalues presented in Ref. [20] vanishes gradually and the number of the branches decreases with the time lapses, but the imaginary part of the eigenvalues shown in Fig. 10 split into five branches till $t=60$ even if the values of the imaginary part decrease slowly with the time lapses, which is another effect of the periodic perturbation term in the NLSE (1).

Inasmuch as no analytical approach available for the conditions for the occurrence of the breakup of the multisoliton state, some numerical experiments on the propagations of other multisolitons of the NLSE (1) with different initial conditions are also performed to explore the conditions for the occurrence of the breakup. Unfortunately, the breakup of the multisoliton state occurs at different levels in all the cases considered in our further experiments, which may imply that the multisoliton state of the NLSE (1) is more likely splitting into individual solitons than that of the NLSE without periodic excitation. The numerical results of those experiments will not be shown to avoid prolixity.

\section{Conclusions}

To explore some dynamic behaviors of the damping NLSE with periodic perturbation, an almost structure-preserving approach focusing on the local energy/momentum losses is employed in this paper. Based on the multi-symplectic order reduction theory, the approximate symmetric form for the damping NLSE, named as generalized multi-symplectic form, is presented with several approximate local conservation laws. Then, a six-point scheme that is equivalent to the Preissmann box scheme for the generalized multi-symplectic form is constructed. Finally, numerical experiments are performed, the purpose of which includes two aspects: one is to verify the structure-preserving properties of the scheme; another is to 
investigate the breakup process of the multisoliton state of the damping NLSE.

The excellent numerical properties of the scheme constructed in this paper have been illustrated by the associated numerical results. To insure the structure-preserving properties of the scheme, the maximum value of the damping rate is obtained with fixed step lengths according to the generalized multi-symplectic definition. With linear damping rate that is less than the threshold, the local energy/momentum losses are recorded and the relative deviations between the certain numerical integrations results of which with different perturbation amplitudes are presented in the numerical experiments, which are agreed with the analytical results presented in Section 2 and imply that the six-point scheme owns good structure-preserving characteristics.

The numerical results on the breakup phenomena of the multisoliton state of the damping NLSE with periodic perturbation illustrate that the breakup phenomena also occur when a periodic perturbation is added to the linearly damping NLSE. Moreover, the existence of the breakup phenomena is independent of the initial conditions, which implies that the external periodic excitation may weaken the stability of the multisoliton state of the linearly damping NLSE.

\section{Acknowledgements}

The authors wish to thank Professors James J. Feng and Tsai, Tai-Peng of the University of British Columbia for giving us several good suggestions. The research is supported by the National Natural Science Foundation of China (11372252, 11372253 and 11432010), the

Science Foundation of Aviation of China (2013ZB53020), 111 project (B07050) to the Northwestern Polytechnical University, the Shaanxi National Science Foundation (2015JM1026), the Astronautics Supporting Technology Foundation of China (2015-HT-XGD), the Fundamental Research Funds for the Central Universities (3102014JCQ01035), the Open Foundation of State Key Laboratory of Structural Analysis of Industrial Equipment (GZ1312) and the fund of the State Key Laboratory of Solidification Processing in NWPU. 


\section{References}

[1] Schrödinger, E. An undulatory theory of the mechanics of atoms and molecules, Physical Review. 28 (1926) 1049-70.

[2] Quintero, NR, Mertens, FG, Bishop, AR. Soliton stability criterion for generalized nonlinear Schrödinger equations, Physical Review E. 91 (2015) 012905.

[3] Levitsky, M, Tangherlini, FR. Schrödinger's radial equation, Physics Today. 68 (2015) 8-9.

[4] Terekhov, IS, Vergeles, SS, Turitsyn, SK. Conditional Probability Calculations for the Nonlinear Schrödinger Equation with Additive Noise, Physical Review Letters. 113 (2014) 230602.

[5] Ablowitz, MJ, Musslimani, ZH. Integrable Nonlocal Nonlinear Schrödinger Equation, Physical Review Letters. 110 (2013) 064105.

[6] Arevalo, E. Soliton Theory of Two-Dimensional Lattices: The Discrete Nonlinear Schrödinger Equation, Physical Review Letters. 102 (2009) 224102.

[7] Belic, M, Petrovic, N, Zhong, WP, Xie, RH, Chen, G. Analytical light bullet solutions to the generalized (3+1)-dimensional nonlinear Schrödinger equation, Physical Review Letters. 101 (2008) 123904.

[8] Shlizerman, E, Rom-Kedar, V. Three types of chaos in the forced nonlinear Schrödinger equation, Physical Review Letters. 96 (2006) 024104.

[9] Kruglov, VI, Peacock, AC, Harvey, JD. Exact self-similar solutions of the generalized nonlinear Schrödinger equation with distributed coefficients, Physical Review Letters. 90 (2003) 113902.

[10] Serkin, VN, Hasegawa, A. Novel soliton solutions of the nonlinear Schrödinger equation model, Physical Review Letters. 85 (2000) 4502-5.

[11] Bergé, L. Wave collapse in physics: principles and applications to light and plasma waves, Physics Reports. 303 (1998) 259-370.

[12] Gutkin, E. Quantum nonlinear Schrödinger equation: Two solutions, Physics Reports. 167 (1988) 1-131.

[13] Ostlund, S, Pandit, R, Rand, D, Schellnhuber, HJ, Siggia, ED. One-dimensional Schrödinger-equation with an almost periodic potential, Physical Review Letters. 50 (1983) 1873-6.

[14] Kosloff, D, Kosloff, R. A fourier Method solution for the time-dependent Schrödinger-equation as a tool in molecular-dynamics, Journal of Computational Physics. 52 (1983) 35-53.

[15] Balian, R, Bloch, C. Solution of Schrödinger equation in terms of classical paths, Annals of Physics. 85 (1974) 514-45.

[16] Kohn, W, Rostoker, N. Solution of the Schrödinger equation in periodic lattices with an application to metallic lithium, Physical Review. 94 (1954) 1111-20.

[17] Caldeira, AO, Leggett, AJ. Quantum tunnelling in a dissipative system, Annals of Physics. 149 (1983) 374-456. 
[18] Kostin, MD. On the Schrödinger - Langevin Equation, The Journal of Chemical Physics. 57 (1972) 3589-91.

[19] Davidson, A. Damping in Schrödinger's equation for macroscopic variables, Physical Review A. 41 (1990) 3395.

[20] Prilepsky, JE, Derevyanko, SA. Breakup of a multisoliton state of the linearly damped nonlinear Schrödinger equation, Physical Review E. 75 (2007) 036616.

[21] Peng, JH, Tang, JS, Yu, DJ, Yan, JR, Hai, WH. Solutions, bifurcations and chaos of the nonlinear Schrödinger equation with weak damping, Chinese Physics. 11 (2002) 213-7.

[22] Christiansen, PL, Gaididei, YB, Johansson, M, Rasmussen, KO, Yakimenko, II. Collapse of solitary excitations in the nonlinear Schrödinger equation with nonlinear damping and white noise, Physical Review E. 54 (1996) 924-30.

[23] Malomed, BA. Soliton-collision problem in the nonlinear Schrödinger-equation with a nonlinear damping term, Physical Review A. 44 (1991) 1412-4.

[24] Bondeson, A, Ott, E, Antonsen, TM. Quasiperiodically forced damped pendula and Schrödinger-equations with quasiperiodic potentials - implications of their equivalence, Physical Review Letters. 55 (1985) 2103-6.

[25] Nicholson, DR, Goldman, MV. Damped nonlinear Schrödinger equation, Physics of Fluids. 19 (1976) 1621-5.

[26] Delfour, M, Fortin, M, Payr, G. Finite-difference solutions of a non-linear Schrödinger equation, Journal of Computational Physics. 44 (1981) 277-88.

[27] Peranich, LS. A finite-difference scheme for solving a nonlinear Schrödinger-equation with a linear damping term, Journal of Computational Physics. 68 (1987) 501-5.

[28] Zhang, FY, Lu, SJ. Long-time behavior of finite difference solutions of a nonlinear Schrödinger equation with weakly damped, Journal of Computational Mathematics. 19 (2001) 393-406.

[29] Zhang, FY. Long-time behavior of finite difference solutions of three-dimensional nonlinear Schrödinger equation with weakly damped, Journal of Computational Mathematics. 22 (2004) 593-604.

[30] Bao, WZ, Jaksch, D. An explicit unconditionally stable numerical method for solving damped nonlinear Schrödinger equations with a focusing nonlinearity, SIAM Journal on Numerical Analysis. 41 (2003) 1406-26.

[31] Asadzadeh, M, Rostamy, D, Zabihi, F. Discontinuous Galerkin and Multiscale Variational Schemes for a Coupled Damped Nonlinear System of Schrödinger Equations, Numerical Methods for Partial Differential Equations. 29 (2013) 1912-45.

[32] Su, HL, Qin, MZ. Symplectic schemes for Birkhoffian system, Communications in Theoretical Physics. 41 (2004) 329-34.

[33] Moore, BE, Norena, L, Schober, CM. Conformal conservation laws and geometric integration for damped Hamiltonian PDEs, Journal of Computational Physics. 232 (2013) 214-33.

[34] Pereira, NR. Soliton in the damped nonlinear Schrödinger equation, Physics of Fluids (1958-1988). 20 (1977) 1735-43. 
[35] Nicholson, DR, Goldman, MV. Damped nonlinear Schrödinger equation, Physics of Fluids (1958-1988). 19 (1976) 1621-5.

[36] Bridges, TJ. Multi-symplectic structures and wave propagation, Mathematical proceedings of the Cambridge Philosophical Society. 121 (1997) 147-90.

[37] Islas, AL, Schober, CM. Multi-symplectic methods for generalized Schrödinger equations, Future Generation Computer Systems. 19 (2003) 403-13.

[38] Sun, JQ, Qin, MZ. Multi-symplectic methods for the coupled 1D nonlinear Schrödinger system, Computer Physics Communications. 155 (2003) 221-35.

[39] Aydin, A, Karasozen, B. Symplectic and multi-symplectic methods for coupled nonlinear Schrödinger equations with periodic solutions, Computer Physics Communications. 177 (2007) 566-83.

[40] Hong, J, Liu, XY, Li, C. Multi-symplectic Runge-Kutta-Nyström methods for nonlinear Schrödinger equations with variable coefficients, Journal of Computational Physics. 226 (2007) 1968-84.

[41] Qian, X, Song, SH, Chen, YM. A semi-explicit multi-symplectic splitting scheme for a 3-coupled nonlinear Schrödinger equation, Computer Physics Communications. 185 (2014) 1255-64.

[42] Zhu, HJ, Song, SH, Tang, YF. Multi-symplectic wavelet collocation method for the nonlinear Schrödinger equation and the Camassa-Holm equation, Computer Physics Communications. 182 (2011) 616-27.

[43] Hu, WP, Deng, ZC, Han, SM, Zhang, WR. Generalized Multi-symplectic Integrators for a Class of Hamiltonian Nonlinear Wave PDEs, Journal of Computational Physics. 235 (2013) 394-406.

[44] Hu, WP, Deng, ZC. Chaos in embedded fluid-conveying single-walled carbon nanotube under transverse harmonic load series, Nonlinear Dynamics 79 (2015) 325-33.

[45] Hu, W, Deng, Z. Competition between geometric dispersion and viscous dissipation in wave propagation of KdV-Burgers equation, Journal of Vibration and Control. 21 (2015) 2937-45.

[46] Hu, WP, Deng, ZC, Wang, B, Ouyang, HJ. Chaos in an embedded single-walled carbon nanotube, Nonlinear Dynamics 72 (2013) 389-98.

[47] Preissmann, A. Propagation des intumescences dans les canaux et rivieres. First Congress French Association for Computation, Grenoble, 1961. pp. 433-42.

[48] Zhao, PF, Qin, MZ. Multisymplectic geometry and multisymplectic Preissmann scheme for the KdV equation, Journal of Physics A-Mathematical and General. 33 (2000) 3613-26.

[49] Laurie, DP. Calculation of Gauss-Kronrod Quadrature Rules, Mathematics of Computation. 66 (1997) 1133-45.

[50] Calvetti, D, Golub, GH, Gragg, WB, Reichel, L. Computation of Gauss-Kronrod Quadrature Rules, Mathematics of Computation. 69 (2000) 1035-52.

[51] Yang, J. Nonlinear Waves in Integrable and Nonintegrable Systems, Society for Industrial and Applied Mathematics; 2010.

[52] Boyd, JP. Chebyshev and Fourier spectral methods, Courier Corporation; 2001. 